\title{
Re-Analysis of the Solar Phase Curves of the Icy Galilean Satellites
}

\author{
Deborah Domingue \\ Lunar and Planetary Institute, 3600 Bay Area Botlevard, Houston, Texas 77058 \\ E-mail: domingue@ilpi.jsc.nasa.gov \\ and \\ Anne Verbiscer ${ }^{1}$ \\ Center for Radiophysics and Space Research, Comell University, Ithaca. New York 14853 \\ Received March 1, 1996; revised Fcbruary 27, 1997
}

\begin{abstract}
Re-analysis of the solar phase curves of the icy Galilean satellites demonstrates that the quantitative results are dependent on the single particle scattering function incorporated into the photometric model; however, the qualitative properties are independent. The results presented here show that the general physical characteristics predicted by a Hapke model (B. Hapke, 1986, Icarus 67, 264-280) incorporating a two parameter double Henyey-Greenstein scattering function are similar to the predictions given by the same model incorporating a three parameter double Henyey-Greenstein scattering function as long as the data set being modeled has adequate coverage in phase angle. Conflicting results occur when the large phase angle coverage is inadequate. Analysis of the role of isotropic versus anisotropic multiple scattering shows that for surfaces as bright as Europa the two models predict very similar results over phase angles covered by the data. Differences arise only at those phase angles for which there are no data. The single particle scattering behavior between the leading and trailing hemispheres of Europa and Ganymede is commensurate with magnetospheric alterations of their surfaces. Ion bombardment will produce more forward scattering single scattering functions due to annealing of potential scattering centers within regolith particles (N. J. Sack et al., 1992, Icarus 100, 534-540). Both leading and trailing hemispheres of Europa are consistent with a high porosity model and commensurate with a frost surface. There are no strong differences in predicted porosity between the two hemispheres of Callisto, both are consistent with model porosities midway between that deduced for Europa and the Moon. Surface roughness model estimates predict that surface roughness increases with satellite distance from Jupiter, with lunar surface roughness values falling midway between those measured for Ganymede and Callisto. There is no obvious variation in predicted surface roughness with hemisphere for any of the Galilean satellites. 1997 Academic Press
\end{abstract}

\footnotetext{
1 Current address: Department of Physics and Astronomy, University of Massachusetts, Amherst, MA 010003 .
}

\section{INTRODUCTION}

The photometric properties of a planetary surface are determined by the physical structure and texture of the surface regolith and its material. Photometric modeling attempts to describe and place constraints on such properties as roughness, porosity, and regolith grain size distribution, while also describing and placing constraints on such material properties as the average single scattering albedo and single scattering functions. All these properties are influenced or determined by the geological processes that formed and shaped the planetary surface. Therefore understanding the photometrically determined physical structure of a planetary reolith provides supporting evidence for many geologic processes such as micrometeorite and ion bombardment. Photometric modeling can also assist in interpretations of spectral data, where such regolith properties as grain size and compositional mixing can influence the shape of reflectance spectra. This makes photometric modeling a useful tool in comparative planetology.

\section{Background}

The solar phase curves of the icy Galilean satellites have been modeled using Hapke's theory (Hapke 1981, 1984, 1986) by several investigators (Buratti 1985, 1991, Domingue et al. 1991. Helfenstein 1986). Buratti (1985) examined Europa's phase curve based on Voyager images using an early version of Hapke's theory. She did not separate her data into leading versus trailing hemispheres, but examined the surface of Europa as a whole. Buratti (1985) concluded from her analyses that the optically active portion of Europa's surface is highly compact and the regolith particles show nearly isotropic scattering behavior. Domingue et al. (1991) expanded on her work by combining telescopic observations with the Voyager image data to 
construct phase curves that included opposition data (that were not available to Buratti) and by separating the data into leading and trailing hemisphere subsets to examine any potential hemispherical dichotomies. Their modeling of the new opposition data with an updated version of Hapkie's theory (Hapke 1986) predicts that the optically active portions of the regolith on both hemispheres of Europa are very porous, consistent with a frost layer. Domingue et al. (1991) found that their results were consistent with the regolith particles of the leading hemisphere being strongly forward scattering and the regolith particles of the trailing hemisphere having both forward and backward scattering components to their scattering function. The differences in the particle scattering behavior found by Buratti (1985) and Domingue et al. (1991) can be attributed to their choices in the single particle scattering function incorporated into Hapke's model. The different compaction states found by these two studies are due to the inclusion of the telescopic data, which measures the opposition effect in more detail and to a smaller phase angle than the Voyager data alone. Helfenstein (1986) applied Hapke's model to individual geologic terrains on Ganymeade using Voyager image data. The terrains on Ganymede can be divided into two principle units, bright and dark. Helfenstein (1986) found that on average the photometric properties, as deduced from Hapke's model, for the dark terrains show a greater macroscopic roughness, a higher state of compaction, a more uniform single scattering behavior, and a lower single scattering albedo than the bright terrains. Buratti (1991) examined the disk-integrated phase curves of both Ganymede and Callisto using a combination of telescopic observations and measurements from the Voyager images. She divided her data sets into leading and trailing hemispheres in order to examine any hemispherical dichotomies in the photometric surface characteristics of these two satellites. Buratti (1991) found that Ganymede displays no hemispherical differences in surface roughness, compaction state of the optically active layer, or scattering behavior of the regolith particles. She attributed these results to the balance of magnetospheric interactions on the trailing hemisphere with micrometeoritic erosion on the leading hemisphere. Buratti (1991) did find hemispherical differences on Callisto where the optically active layer of the trailing hemisphere's regolith is more compact than the leading hemisphere's regolith and the leading hemisphere regolith particles are more backscattering than those on the trailing hemisphere.

The research presented here is a reexaminationof the disk-integrated phase curves of all three of the icy Galilean satellites. The phase curves examined are constructed from combinations of telescopic observations and Voyager image measurements, following the methods of Buratti (1991) and Domingue et al. (1991). New, previously unpublished, telescopic observations by Lockwood and Thompson (pri- vate communication) are included for Callisto. All of the studies discussed above assumed the multiply scattered component of surface reflected light is isotropic. This is not a valid assumtion for very bright objects, such as Europa (Mishchenko 1994, Goguen 1996, Verbiscer and Helfenstein 1997). Thus the purpose of this study is twofold: first, it examines the effect of the choice of which single particle scattering function is incorporated into the basic Hapke model where isotropic multiple scattering is assumed. The goal is to examine if the hemispherical differences in surface structure and texture measured by Buratti (1991) and Domingue et al. (1991) are real or an artifact of the choice in single particle scattering function. Second, this paper explores the differences between Hapke modeling results for isotropic and anisotropic modeling of the multiple scattering component for bright objects such as Europa and Ganymede.

\section{Model}

An integral part of any photometric theory which attempts to model scattering from a planetary regolith is the incorporation of a single particle scattering function. The single particle scattering function's role is to describe how an idealized, average particle or grain from a planetary regolith scatters light. Such knowledge is an important verification tool for various geologic processes. In Hapke's model it is assumed that all regolith particles or grains are large compared to the wavelength of incident light. McGuire and Hapke (1995) studied the changes in the single particle scattering function as a function of particle shape, texture, and clarity. Using 1-cm (in diamter) sized artificial resin particles McGuire and Hapke measured the scattering functions as shape, particle surface texture, and density of internal scattering material were systematically varied for incident wavelengths of 448,554 , and $690 \mathrm{~nm}$. They found that to model the resulting scattering phase curves of their artificial particles a two parameter single particle scattering function was needed and that a two parameter double Henyey-Greenstein function modeled their results better than a two parameter Legendre polynomial. Hartman and Domingue (1997) compared the quality of fit between a two parameter double Henyey-Greenstein function versus a three parameter double HenyeyGreenstein function to the McGuire and Hapke (1995) scattering data. The goal of their research was to establish which type of scattering function was essential for obtaining physically meaningful results from Hapke's model. Helfenstein et al. (1997) also fit the McGuire and Hapke (1995) scattering data with a three parameter HenyeyGreenstein function to examine the relationship between scattering albedo and single scattering function. The double Henyey-Greenstein functions are defined and discussed under Analytical Methods. In the course of their 
study Hartman and Domingue (1997) found that the choice of single particle scattering function influenced the values of the non-opposition Hapke parameters (the opposition Hapke parameters are those which describe the opposition effect, all others are non-opposition parameters).

In the first set of analyses presented here the phase curve data are modeled twice, once incorporating a two parameter double Henyey-Greenstein function and then a second time incorporating a three parameter double Henyey-Greenstein function into Hapke's model. This differs from the work done by Buratti $(1985,1991)$ in that she used a single parameter Henyey-Greenstein function. Domingue et al. (1991) used a two parameter double Henyey-Greenstein function; therefore, this work will expand on their findings with the three parameter scattering function application. The goal of this initial study is to examine if the hemispherical dichotomies observed in the phase curve analysis of these satellites is a function of the choice in single particle scattering function. This first set of analyses assumes that the multiply scattered component is isotropic.

The second set of analyses investigates the differences in modeling results if anistropic multiple scattering is incorporated into the model. For the second part of this study the phase curves were reanalyzed using a three parameter double Henyey-Greenstein function where it is assumed that the anisotropy in the singly and multiply scattered components can be described by the same scattering function (Verbiscer 1991). The anisotropic multiple scattering analyses use the method described by Verbiscer (1991).

\section{DATA}

\section{Earth-Based Observations}

The Earth-based telescopic observations used in this study are taken from Domingue et al. (1991) for Europa (which are the observations of G. W. Lockwood and D. T. Thompson), from Millis and Thompson (1975) for Ganymede, and from Lockwood and Thompson (private communication) for Callisto. The Europa and Callisto telescopic observations were taken in the Stromgren $y$ and $b$ filters, which have effective wavelengths of 0.55 and 0.47 $\mu \mathrm{m}$, respectively. The Ganymede observations from Millis and Thompson (1975) were taken in the $V$ and $B$ filters, which correspond to 0.55 and $0.44 \mu \mathrm{m}$, respectively. All telescopic data were corrected for rotational phase variations. The rotational phase angle ranges for the leading and trailing hemispheres of all three satellites are listed in Table I. The broad rotational phase angle ranges make any measured leading/trailing hemisphere dichotomies dependent on the rotational phase curve corrections applied to the data sets. The leading hemisphere of Europa was corrected to $95^{\circ}$ rotational phase and the trailing hemisphere was corrected to $295^{\circ}$ rotational phase using the
TABLE I

\begin{tabular}{|c|c|c|c|c|c|}
\hline Satellite & $\begin{array}{c}\text { Data } \\
\text { source }\end{array}$ & $\begin{array}{c}0.47-\mu \mathrm{m} \\
\text { lcad }\end{array}$ & $\begin{array}{c}0.47-\mu \mathrm{m} \\
\text { trail }\end{array}$ & $\begin{array}{c}0.5 .5-\mu \mathrm{ml} \\
\text { lead }\end{array}$ & $\begin{array}{c}0.55-\mu \mathrm{m} \\
\text { trail }\end{array}$ \\
\hline \multicolumn{6}{|c|}{ Satellite phase angle coverage } \\
\hline \multirow[t]{2}{*}{ Europa } & Telescopic & $0.2-11.3$ & $0.2-11.3$ & $0.2-11.3$ & $0.2-11.3$ \\
\hline & Voyager & $3.8-119.0$ & $3.0-119.0$ & $3.7-104.4$ & $2.9-94.3$ \\
\hline \multirow[t]{2}{*}{ Ganymede } & Telescopic & $0.6-11.4$ & $1.4-11.2$ & $0.4-11.4$ & $1.4-11.2$ \\
\hline & Voyager & $4.8-120.3$ & $20.0-124.0$ & $4.9-32.2$ & $20.0-38.7$ \\
\hline \multirow[t]{2}{*}{ Callisto } & Telescopic & $0.4-11.1$ & $0.6-11.2$ & $0.4-11.1$ & $0.6-11.2$ \\
\hline & Voyager & $8.3-50.4$ & $13.9-140.8$ & $8.3-51.7$ & $14.0-107.4$ \\
\hline \multicolumn{6}{|c|}{ Satcllite rotational angle coverage } \\
\hline \multirow[t]{2}{*}{ Europa } & Telescopic & $31-126$ & $213-302$ & $31-126$ & $213-302$ \\
\hline & Voyager & $7-165$ & $2017-3.51$ & $11-162$ & $207-346$ \\
\hline \multirow[t]{2}{*}{ Ganymede } & Telescopic & $12-151$ & $213-.3 .37$ & $12-151$ & $213-.337$ \\
\hline & Voyager & $17-176$ & $185-359$ & $17-176$ & $186-360$ \\
\hline \multirow[t]{2}{*}{ Callisto } & Telescopic & $32-133$ & $226-.312$ & $32-1.33$ & $226-312$ \\
\hline & Voyager & $3-174$ & $184-359$ & $3-171$ & $184-3.52$ \\
\hline
\end{tabular}

Note. Lead corresponds to leading hemisphere. and trail corresponds to trailing hemisphere. All angle ranges are in degrees.

rotational phase curves of Domingue et al. (1991). The reduction of the Europa data follows that of Domingue et al. (1991). The leading hemispheres of Ganymede and Callisto were corrected to $90^{\circ}$ rotational phase angle, whereas the trailing hemispheres of these two satellites were corrected to $270^{\circ}$ rotational phase angle. The corrections applied to Ganymede were based on the rotational phase curves of Millis and Thompson (1975). The corrections applied to Callisto were based on the rotational phase curve observations of Lockwood and Thompson (private communication), Millis and Thompson (1975), and Morrison et al. (1974). Figure 1 shows the correction curve to $255^{\circ}$ rotational phase angle for Callisto. The rotational phase curves for Callisto showed the largest variation between different data sets. The best agreement was between the Millis and Thompson (1975) and the Lockwood and Thompson Callisto data sets. For those regions of the phase curve where there were Lockwood and Thompson measurements, the rotational phase curve corrections based on their data were used. Outside of those regions the phase curve corrections based on the Millis and Thompson data were used. These corrections were used since the telescopic observations in the solar phase curves is predominately the Lockwood and Thompson measurements. The telescopic data, which were corrected to opposition. were also normalized to a Lambert disk of the same size at the same distance according to the relation

$$
M=M_{\text {plinct }}-M_{\text {sun }}-5 \log [(s \delta) /(\Delta r)] .
$$

where $s$ is the Earth-satellite distance (4.2 AU), $\delta$ is the Sun-satellite distance (5.2 AU, Burns 1986). $\Delta$ is the SunEarth distance, and $r$ is the satellite radius. $M_{\text {planer }}$ is the 


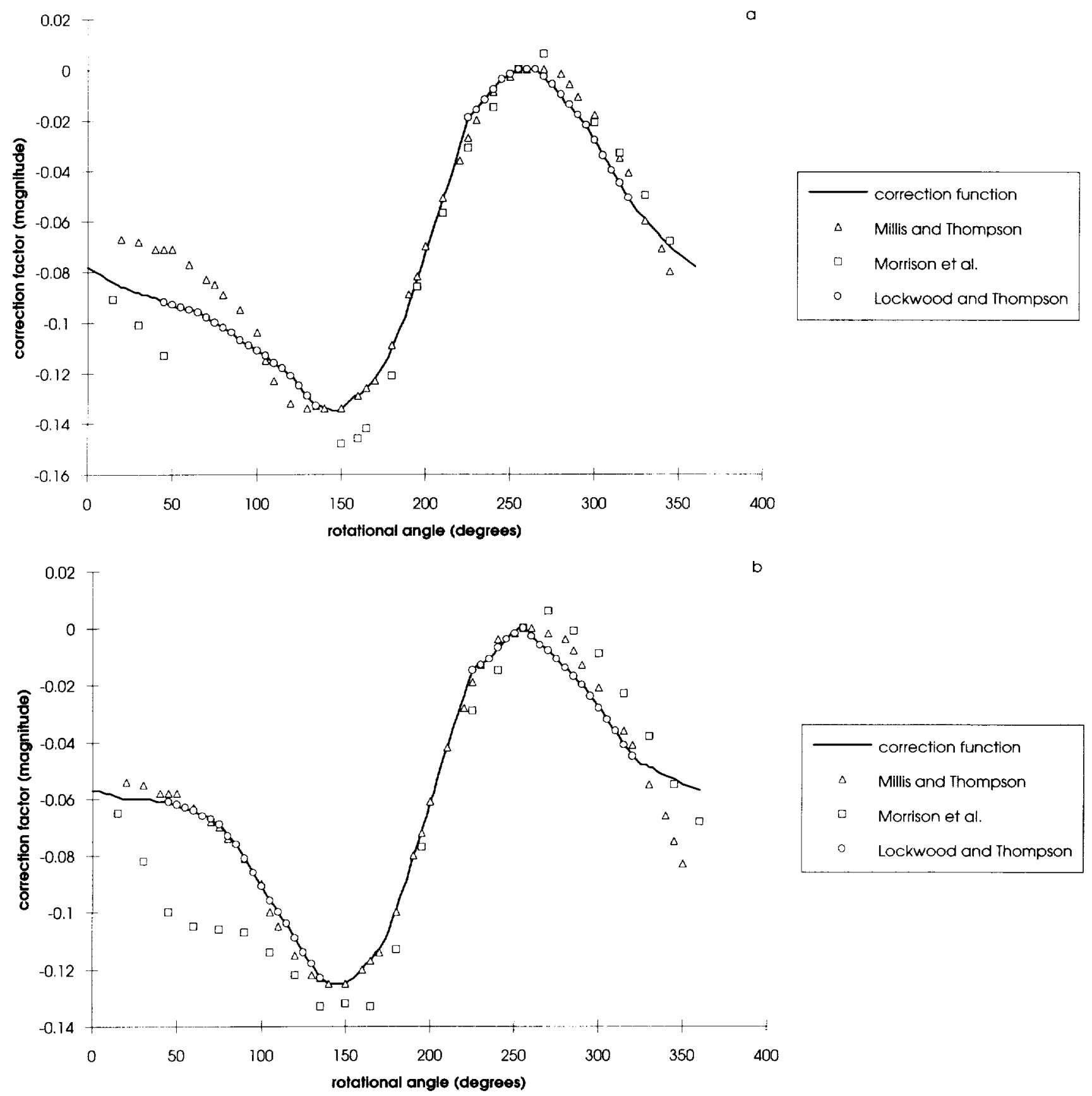

FIG. 1. The correction curves (solid line) used to correct the telescopic and Voyager Callisto data sets for brightness variations due to rotational phase. The corrections are to $255^{\circ}$ rotational phase angle at (a) $0.47 \mu \mathrm{m}$ and (b) $0.55 \mu \mathrm{m}$ and are based on the observations of Lockwood and Thompson (private communication), Millis and Thompson (1975), and Morrison et al. (1974), corresponding to the circles, triangles, and squares, respectively.

measured magnitude of the satellite and $M_{\text {Sun }}$ is the magnitude of the Sun at the same observing wavelength $(4.378$ and 3.728 at 0.448 and $0.55 \mu \mathrm{m}$, respectively; Allen 1973).

\section{Voyager Image Data}

The Voyager images used in this study were taken in the clear, blue, orange, and green filters. It should be noted that Buratti (1991) used only clear filter images in her study of Ganymede and Callisto. All the images used were calibrated using the Planetary Image and Cartography System (PICS), which incorporates the calibration corrections described by Danielson et al. (1991). Since the calibration of these images the correction factors have been updated (E. Eliason and G. Danielson, private communication). 
The use of the older calibration factors produces a $7-8 \%$ uncertainty in the green and orange filter data and an $\sim 3 \%$ uncertainty in the clear and blue filter data. The calibrated images were converted into disk integrated values by averaging the brightness values of the pixels composing the satellite disk. This methods introduces a $\pm 1 \%$ error in the integral data. The intensity measurements were then converted to magnitudes to correspond with the telescopic observations.

The average brightness values were corrected for rotational phase angle variations using the rotational phase curve corrections applied to the telescopic data for the same satellite. The Voyager longitude system differs from the longitude system utilized by astronomers by $1^{\circ}$ or $2^{\circ}$. This small difference may introduce small error bars in the rotational corrections. Images where the subspacecraft longitude fell between $0^{\circ}$ and $180^{\circ}$ were grouped into the leading hemisphere data set. and similarly, the images where the subspacecraft longitude fell between $180^{\circ}$ and $360^{\circ}$ were grouped into the trailing hemisphere data set. This method poses a potential problem at high phase angles where the subspacecraft longitude is not centered on its respective hemisphere. The images used here have been examined for data points which have been binned in one hemisphere category but actually have the illuminated portion of their disk in the other hemisphere. For the images in this study this method bins the images correctly, where the illuminated portion of the disk at high phase angles is $50 \%$ of the hemisphere it has been assigned. Table I lists the range of rotational and solar phase angles for the satellites.

Images were taken by two spacecraft (Voyagers I and II), each having a narrow and a wide-angle camera. Within each spacecraft image set comparisons at similar solar phase angles within each filter were made between narrow angle camera images and wide angle camera images. A correction was applied to the wide angle camera brightness values to match them to the narrow angle camera brightness values if they did not coincide. The Voyager II clear and blue filter wide angle camera images used in the Europa phase curves required a correction factor of -0.13 magnitudes, whereas the Voyager II orange and green filter wide angle camera images for the Europa phase curves required a -0.036 magnitude correction. The Ganymede wide angle camera images from both spacecraft required no corrections. The Callisto clear filter images from both spacecraft also required no wide angle camera corrections. However, the Voyager I blue filter wide angle camera images required a -0.198 magnitude correction, whereas the same Voyager II images needed a 0.145 magnitude correction. The corrections for the Callisto orange and green filter images were more complicated. The Voyager II wide angle camera images for these two filters required corrections of -0.154 and -0.178 magnitudes, respectively. The Voyager I wide angle camera images required correc- tions of -0.088 and -0.178 magnitudes for these two filters. respectively, for the leading hemisphere images. The trailing hemisphere images required corrections of $0.775 \mathrm{mag}$ nitudes for both filters. This dependence on hemisphere may be related to errors in the rotational phase angle corrections.

The next correction to the Voyager brightness values was for wavelength. The effective wavelengths of the clear and orange filters are 0.47 and $0.55 \mu \mathrm{m}$, respectively. This corresponds to the wavelengths of the telescopic observations. The blue and green Voyager filters have effective wavelengths of 0.48 and $0.58 \mu \mathrm{m}$, respectively. A wavelength correction was applied to the blue filter data to match it with the clear filter data by comparing the brightness values from these images at overlapping solar phase angles. A similar correction was applied to the green filter data to match it with the orange filter data. The filter corrections for the Europa phase curves were -0.104 and -0.074 magnitudes for the Voyager I blue filter images for the leading and trailing hemispheres, respectively. The corresponding Voyager II corrections were 0.076 and 0.092 magnitudes. The filter corrections for the Europa phase curves for the orange filter Voyager I images were 0.015 magnitudes for the trailing hemisphere, and no correction was applied to the leading hemisphere orange filter images. No corrections for wavelength were applied to the images used in the Ganymede phase curves. The corrections to the blue filter images for the Callisto phase curve measurements were 0.029 and 0.041 for the Voyager I and II images. respectively. Similarly for the Callisto orange filter images the corrections were 0.028 and -0.015 for Voyagers I and II, respectively.

Once the camera corrections were applied then a similar comparison was made between the two spacecraft data sets for each filter. The Voyager I images were corrected to coincide with the Voyaer II images where necessary. These corrections consisted of -0.008 and -0.115 magnitude corrections to the $0.55-\mu \mathrm{m}$ (orange and green) Europa phase curves for the leading and trailing hemispheres, respectively. The corresponding corrections for the $0.47-\mu \mathrm{m}$ (clear and blue) Europa phase curves were 0.082 and 0.193 . respectively. The correction to the Voyager I Ganymede images was 0.06 for both hemispheres and wavelengths. The Callisto phase curves required a correction of only 0.125 to the Voyager II $0.47-\mu \mathrm{m}$ images to bring them into correspondence with the Voyager I images.

The final correction to the Voyager data was made by comparing it to the corresponding telescopic observations. The clear and blue filter data were corrected to match the $0.47-\mu \mathrm{m}$ telescopic data (in the case of Ganymede the telescopic observations were at $0.44 \mu \mathrm{m}$ ) by comparing the brightness values at overlapping solar phase angles. Similarly, the orange and green filter data were corrected to match the $0.55-\mu \mathrm{m}$ telescopic data. These corrections 
for Europa's phase curves at $0.47 \mu \mathrm{m}$ were -0.259 and -0.317 magnitudes for the leading and trailing hemispheres, respectively. The corresponding corrections to the Europa $0.55-\mu \mathrm{m}$ phase curves were -0.124 and -0.141 magnitudes, respectively. For Ganymede the corrections were -0.12 and -0.14 magnitudes for both hemispheres at 0.47 and $0.55 \mu \mathrm{m}$, respectively. These corrections for Callisto's phase curves at $0.47 \mu \mathrm{m}$ were -0.17 and -0.253 magnitudes for the leading and trailing hemispheres, respectively, and were -0.07 and -0.157 magnitudes for Callisto's phase curves at $0.55 \mu \mathrm{m}$ for the leading and trailing hemispheres, respectively.

\section{ANALYTICAL METHODS}

In the first set of analyses concerning the affect of the choice of single particle scattering function. Hapke's model (Hapke 1981, 1984, 1986) was fit to the data using a modified least squares grid search. Each data set was modeled twice, once using Hapke's model with a two parameter double Henyey-Greenstein function (2P-HG function) and a second time using a three parameter double HenyeyGreenstein function (3P-HG function). Isotropic multiple scattering was assumed. The form of the $2 \mathbf{P}-\mathrm{HG}$ function used is given by

$$
\begin{aligned}
P_{2}(\alpha)= & \left(\left(1-c_{2}\right)\left(1-b_{2}^{2}\right) /\left[1+2 b_{2} \cos (\alpha)+b_{2}^{2}\right]^{3 / 2}\right) \\
& +\left(c_{2}\left(1-b_{2}^{2}\right) /\left[1-2 b_{2} \cos (\alpha)+b_{2}^{2}\right]^{3 / 2}\right)
\end{aligned}
$$

where $\alpha$ is the phase angle, and $b_{2}$ and $c_{2}$ are the single particle scattering parameters. The form of the $3 \mathrm{P}-\mathrm{HG}$ function used is given by

$$
\begin{aligned}
P_{3}(\alpha)= & \left(\left(1-c_{3}\right)\left(1-b_{3}^{2}\right) /\left[1+2 b_{3} \cos (\alpha)+b_{3}^{2}\right]^{3 / 2}\right) \\
& +\left(c_{3}\left(1-d_{3}^{2}\right) /\left[1-2 d_{3} \cos (\alpha)+d_{3}^{2}\right]^{3 / 2}\right),
\end{aligned}
$$

where $\alpha$ is the phase angle, and $b_{3}, c_{3}$, and $d_{3}$ are the single particle scattering parameters. Note that the $3 \mathrm{P}-\mathrm{HG}$ function defaults to the $2 \mathrm{P}-\mathrm{HG}$ function for cases, where $d_{3}=-b_{3}$. The results of fitting Hapke's isotropic multiple scattering model to our data sets are listed in Table II along with the corresponding RMS values. The RMS values are defined such that

$$
\mathrm{RMS}=\left\{\left[\sum\left(M_{n}-m_{n}\right)^{2}\right] / N\right\}^{1 / 2},
$$

where $N$ is the number of observational data points, $M$ is the calculated magnitude from Hapke's model, $m$ is the observed magnitude, and the sum is over the number of points. Figures 2 through 7 plot our theoretical results versus the data sets.

In the second set of analyses Hapke's model was fit to the phase curves of Europa and Ganymede using the method of Verbiscer (1991), which incorporates anisotropic multiple scattering. This method assumes that the anisotropy in the single and multiple scattered components is described by the same scattering function. The $3 \mathrm{P}-\mathrm{HG}$ function was used. The results are listed in Table III and the corresponding phase curves are plotted in Figs. 2 through 7. Only the $0.47-\mu \mathrm{m}$ Ganymede data were fit since it has adequate large $\left(>90^{\circ}\right)$ phase angle coverage. The Callisto phase curves were not modeled since its surface is darker than Europa's or Canymede's; thus the role of multiple scattering is weaker. Anisotropic multiple scattering will be most important for bright surfaces, therefore any differences in isotropic versus anisotropic multiple scattering will be most evident in the modeling of the brighter surfaces of Europa and Ganymede.

\section{ERROR ANALYSIS}

Error analysis based on the data set and on the mathematical inter-relationship of the Hapke model parameters is complicated and non-trivial. The value, and changes in the value, of each parameter affects the shape of the phase curve in different phase angle ranges. Changes in the shape of the phase curve due to changes in the value of one parameter oftentimes may be countered by changes in another parameter. Therefore, while an error analysis begins with studying the affects of the individual parameters on the shape of the phase curve, the more convoluted variations in phase curve shape due to changes in multiple parameters must eventually be considered and evaluated. Below is a description of the evaluation of the error bars on the Hapke parameter values for each satellite. These error bars are summarized in Table IV.

A first order analysis was accomplished by comparing the phase curve of a standard set of Hapke parameters to phase curves where each parameter has been varied individually while the others have been kept constant in order to observe the variations in the phase curve due to the change in that one parameter. It can be shown that the value of the single scattering albedo, $w$, affects the entire range of phase angles, whereas the opposition parameters bo (amplitude) and $h$ (width) affect the shape of the phase curve from $0^{\circ}$ to $30^{\circ}$ solar phase angle. The surface roughness parameter, $\theta$-bar, affects the shape of the phase curve most dramatically at solar phase angles greater than $90^{\circ}$; however, it can influence the shape of the phase curve at phase angles as small as $20^{\circ}$. The affects of the single particle scattering function also span the entire range of phase angles where the values of the single scattering function parameters affect different ranges of phase angle depending on the scattering function chosen. Initial error bars are set by comparing the variation between two phase curves, where the value of one parameter has been 
TABLE II

Hapke Parameters for the Icy Galilean Satellites and Rhea

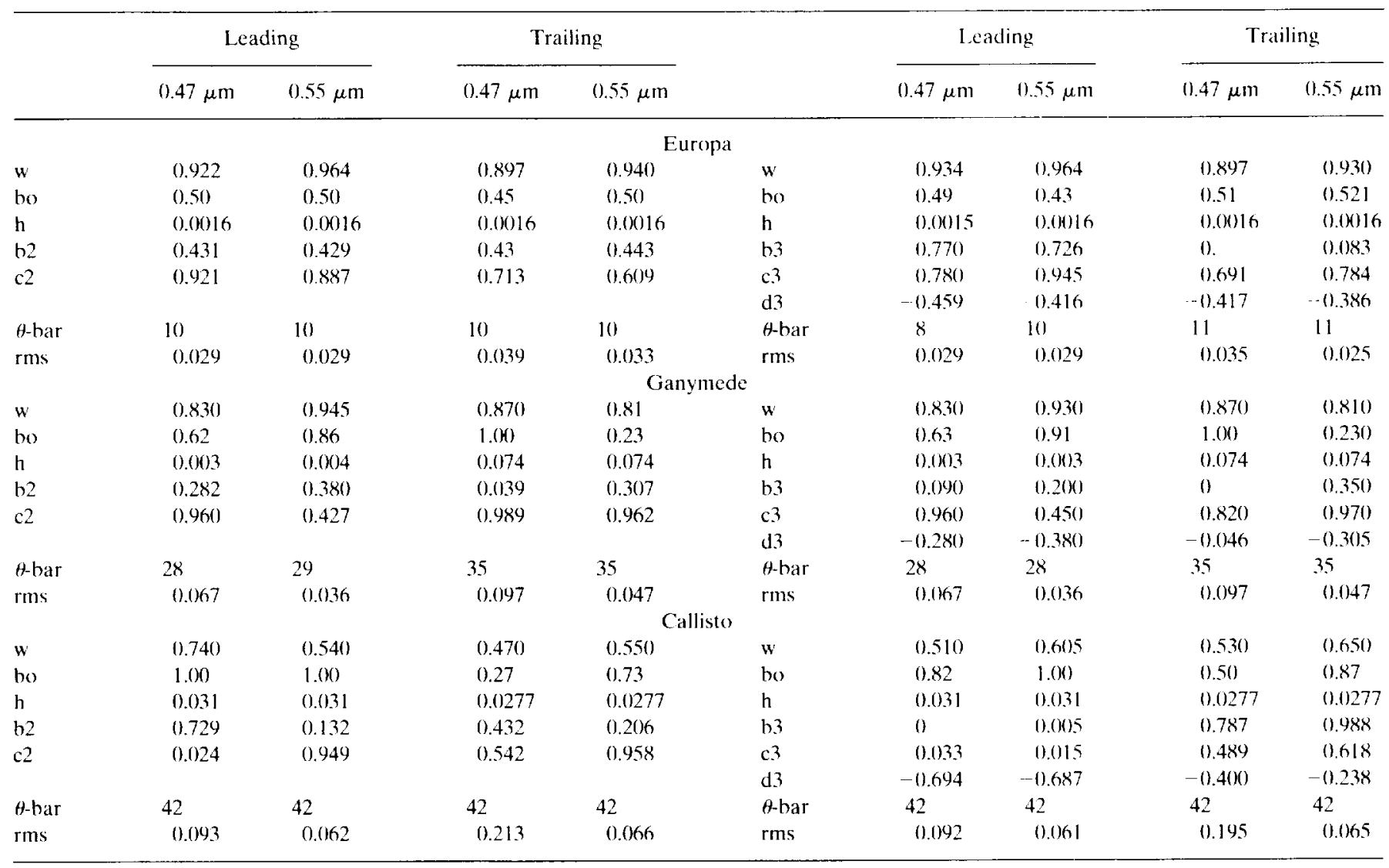

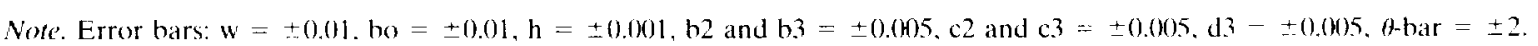

varied, with the scatter and phase angle coverage in the data sets.

The initial error bars for the parameters $w$, bo, and $h$ are $\pm 0.02, \pm 0.1, \pm 0.002$, respectively, for all three of the icy Galilean satellites. The initial error bars for $\theta$-bar are determined by the presence of large phase angle data. If data exist beyond phase angles of $100^{\circ}$ the initial error bars are $\pm 5^{\circ}$. If the large phase angle data exists only out to $70^{\circ}$ the initial error bars are $\pm 10^{\circ}$, and similarly, if the phase angle coverage exists only out to $50^{\circ}$ the initial error bars are $\pm 15^{\circ}$. The Europa the initial error bars in $\theta$-bar are $\pm 5^{\circ}$ for the leading hemisphere in both wavelengths and for the trailing hemisphere at $0.47 \mu \mathrm{m}$. Using these criteria the initial error bar for Europa's trailing hemisphere at $0.55 \mu \mathrm{m}$ is $\pm 10^{\circ}$. However, the surface roughness parameter should be independent of wavelength; therefore the error bar on this parameter for Europa's trailing hemisphere at $0.55 \mu \mathrm{m}$ is also constrained by the modeling results to the $0.47-\mu \mathrm{m}$ data for this hemisphere. Therefore the error bar is less than $\pm 10^{\circ}$ and closer to $\pm 5^{\circ}$. The initial error bars for the $0.47-\mu \mathrm{m}$ data for both hemispheres of Ganymede are $\pm 5^{\circ}$, since there is a data point at $120^{\circ}$ phase angle and the scatter in the large phase angle measurements of the other satellites correlate to $5^{\circ}$ of variaiton in $\theta$-bar. For the $(0.55-\mu \mathrm{m}$ Ganymede data sets the initial error bars based on the range of large phase angle data are $\pm 15^{\circ}$. However, since $\theta$-bar is independent of wavelength, the modeling results of the $0.47-\mu \mathrm{m}$ data constrain the modeling solutions to the $0.55-\mu \mathrm{m}$ data (and vice versa). Therefore the error bars for the Ganymede 0.55 $\mu \mathrm{m}$ data are closer to $\pm 10^{\circ}$ than $\pm 15^{\circ}$. The initial error bars for $\theta$-bar for Callisto are $\pm 5^{\circ}$ for the trailing hemisphere data at both wavelengths and $\pm 15^{\circ}$ for the leading hemisphere in both wavelengths, based on the phase angle coverage in the data sets.

In the analyses presented in this paper two different single particle scattering functions were used: the two parameter double Henyey-Greenstein function (2P-HG), with parameters $b_{2}$ and $c_{2}$, and the three parameter double Henyey-Greenstein function (3P-HG), with parameters $b_{3}, c_{3}$, and $d_{3}$. The 2P-HG function assumes that the halfwidth of the forward and back scattering lobes are equivalent and that the relative amplitude varies. The parameter $b_{2}$ is the width parameter and the parameter $c_{2}$ is the 

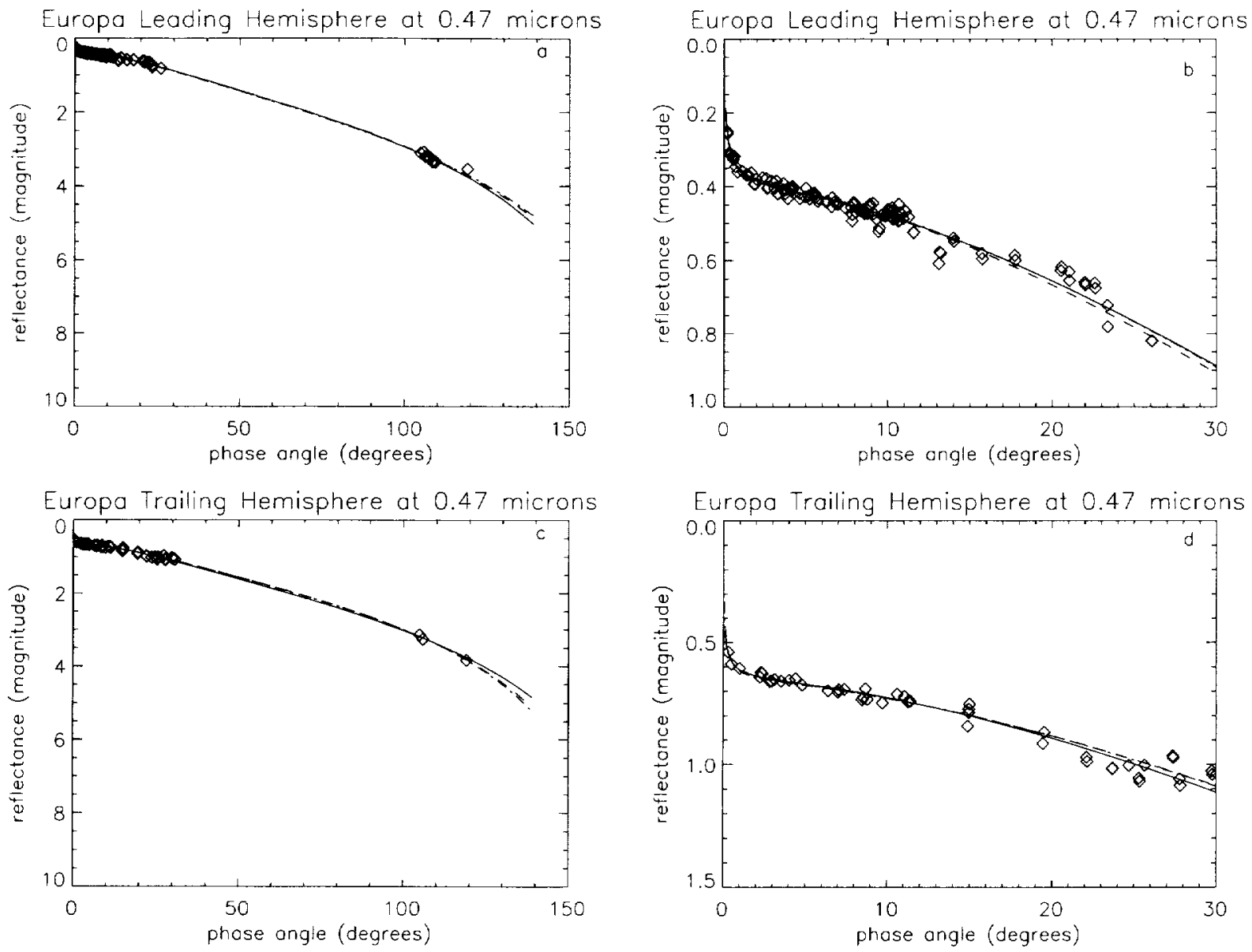

FIG. 2. The solar phase curves of Europa's leading ( $a$ and $b$ ) and trailing ( $c$ and $d$ ) hemispheres at $0.47 \mu \mathrm{m}$, where the diamonds represent the disk-integrated observations, the solid line represents the isotropic multiple scattering Hapke model solution incorporation a two parameter HG function, the dashed line represents the isotropic multiple scattering Hapke model solution incorporating a three parameter HG function, and the dash-dot line represents the anisotropic multiple scattering Hapke model solution incorporation a three parameter HG function. Those places where only a solid line is seen indicates that the solutions lie on top of each other and. therefore, cannot be distinguished.

amplitude parameter. Variations in each of these parameters affects the shape of the phase curve at all phase angles. Any changes at large phase angles, where there are fewer data to constrain the phase curves studied here, also result in changes at low phase angles which is well constrained by the data sets in this study. While changes in the shape of the phase curve due to variations in the values of $b_{2}$ and $c_{2}$ can often by negated by changes in the values of $\theta$-bar and the opposition parameters, for a given set of $b_{2}$ and $c_{2}$ there is a correlating set of unique values for $\theta$-bar and the opposition parameters (and vice versa). Using the method described above the initial error bars for $b_{2}$ and $c_{2}$ for each of the icy Galilean satellites are \pm 0.02 .

The second single particle scattering function used in this study is the three parameter double Henyey-Greenstein function (3P-HG), where $b_{3}$ is the half-width of the forward scattering component, $d_{3}$ is the half-width of the backscat- tered component, and $c_{3}$ is the relative amplitude of these two scattering components. The values of $b_{3}$ are constrained by the high phase angle $\left(\alpha>90^{\circ}\right)$ data and the values of $d_{3}$ are constrained by the low phase angle ( $\alpha<$ $90^{\circ}$ ) data. The value of $c_{3}$ affects the shape of the phase curve over the entire range of phase angles. Changes at large phase angles due to variations in the values of $b_{3}$ or $c_{3}$ can oftentimes be negated by changes in the value of $\theta$-bar depending on the magnitude of the variations in the scattering function parameters. Changes in the shape of the phase curve at small phase angles due to changes in the values of $d_{3}$ or $c_{3}$ can sometimes be compensated for by changes in the opposition parameters bo and $h$. Using the same methods as above the initial error bars for $d_{3}$, and $c_{3}$ are \pm 0.01 and \pm 0.02 , respectively, for the all three of the icy Galilean satellites. The initial error bars for $b_{3}$ depend on the presence and extent of the large phase angle 

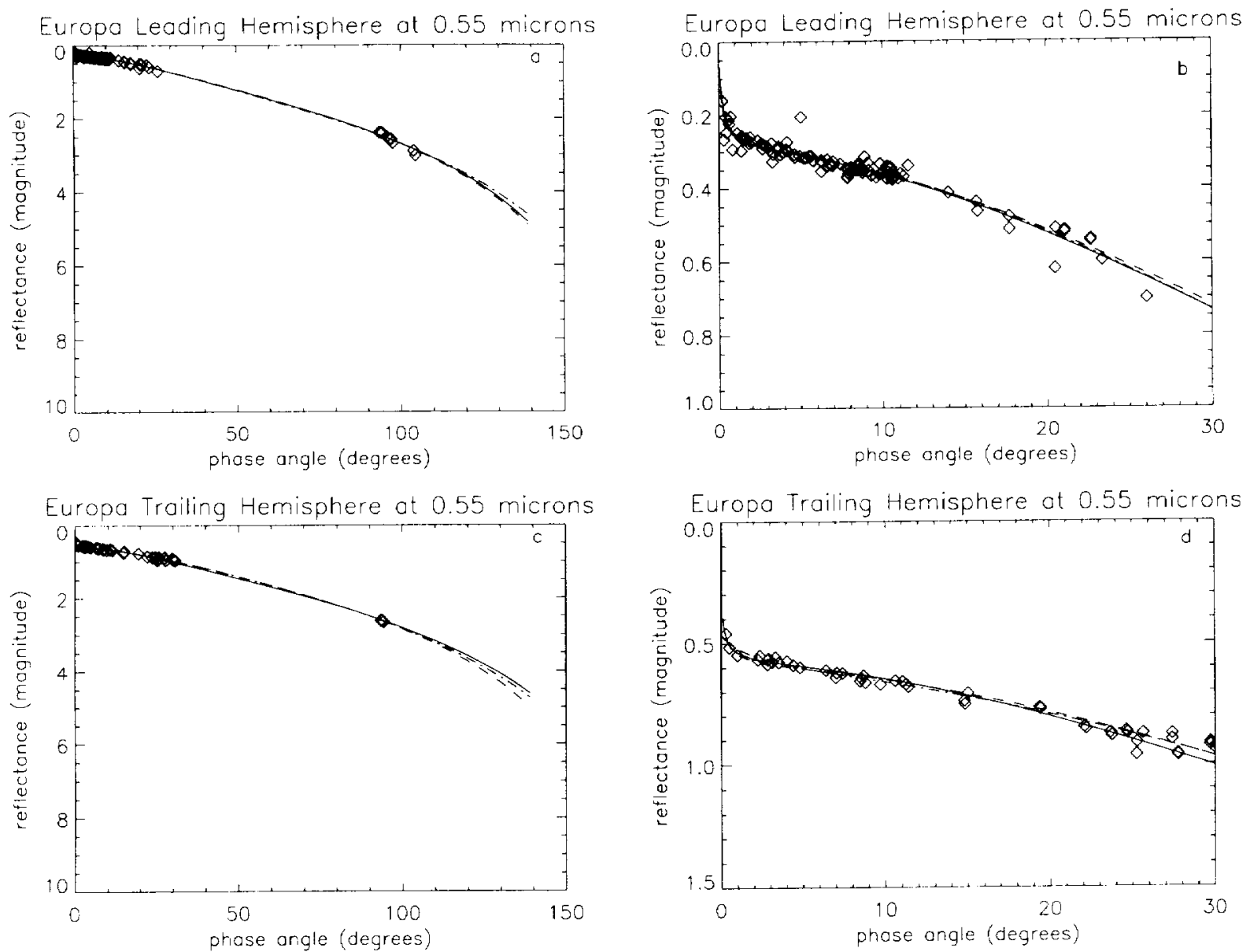

FIG. 3. The solar phase curves of Europa's leading (a and b) and trailing (c and $d$ ) hemispheres at $0.55 \mu \mathrm{m}$. where the diamonds represent the disk-integrated observations, the solid line represents the isotropic multiple scattering Hapke model solution incorporating a two parameter HG function, the dashed line represents the isotropic multiple scattering Hapke model solution incorporating a three parameter HG function, and the dash-dot line represents the anisotropic multiple scattering Hapke model solution incorporating a three parameter HG function. Those places where only a solid line is seen indicates that the solutions lie on top of each other and, therefore. cannot be distinguished.

data. While the values for $\theta$-bar also depend on large phase angle data, $\theta$-bar has the added constraint that it is independent of wavelength. The parameter $b_{3}$, however, does vary with wavelength. Therefore the initial error bars for $b_{3}$ are \pm 0.05 for the leading hemisphere of Europa at both wavelengths, the trailing hemisphere of Europa at 0.47 $\mu \mathrm{m}$, both hemispheres of Ganymede at $0.47 \mu \mathrm{m}$, and the trailing hemisphere of Callisto at both wavelengths. The initial error bars for $b_{3}$ for Europa's trailing hemisphere at $0.55 \mu \mathrm{m}$ is \pm 0.1 and is \pm 0.5 for both hemispheres of Ganymede at $0.55 \mu \mathrm{m}$ and Callisto's leading hemisphere at both wavelengths.

Further constraints on the error bars of the Hapke parameters can be placed by understanding the physical nature of the parameters. The parameter $\theta$-bar is a measure of surface roughness (the tilt of the surface averaged over resolutions ranging from several particle sizes to the resolution of the detector), which is a physical characteristic independent of wavelength. By analyzing the phase curves of the same surface at different wavelengths this parameter can be better constrained and its value can be decoupled from the values of the other parameters in Hapke's model. The value of $\theta$-bar is mathematically coupled to the values of $w$ and the single particle scattering function. Any method of analysis which decouples their affect on the shape of the phase curve also affects the error analysis. The comparison of the disk-integrated solutions to the parameters based on the disk-integrated phase curves to disk-resolved measurements of the same surface is an additional method for decoupling the value of $\theta$-bar, $w$, and the single particle scattering function parameters. By using the disk-integrated parameter solutions and applying their values to disk-resolved data and using the wavelength independent nature of $\theta$-bar, the error bars on $w$ can be constrained to \pm 0.01 based on the scatter in the disk-resolved data for all the icy Galilean satellites. 

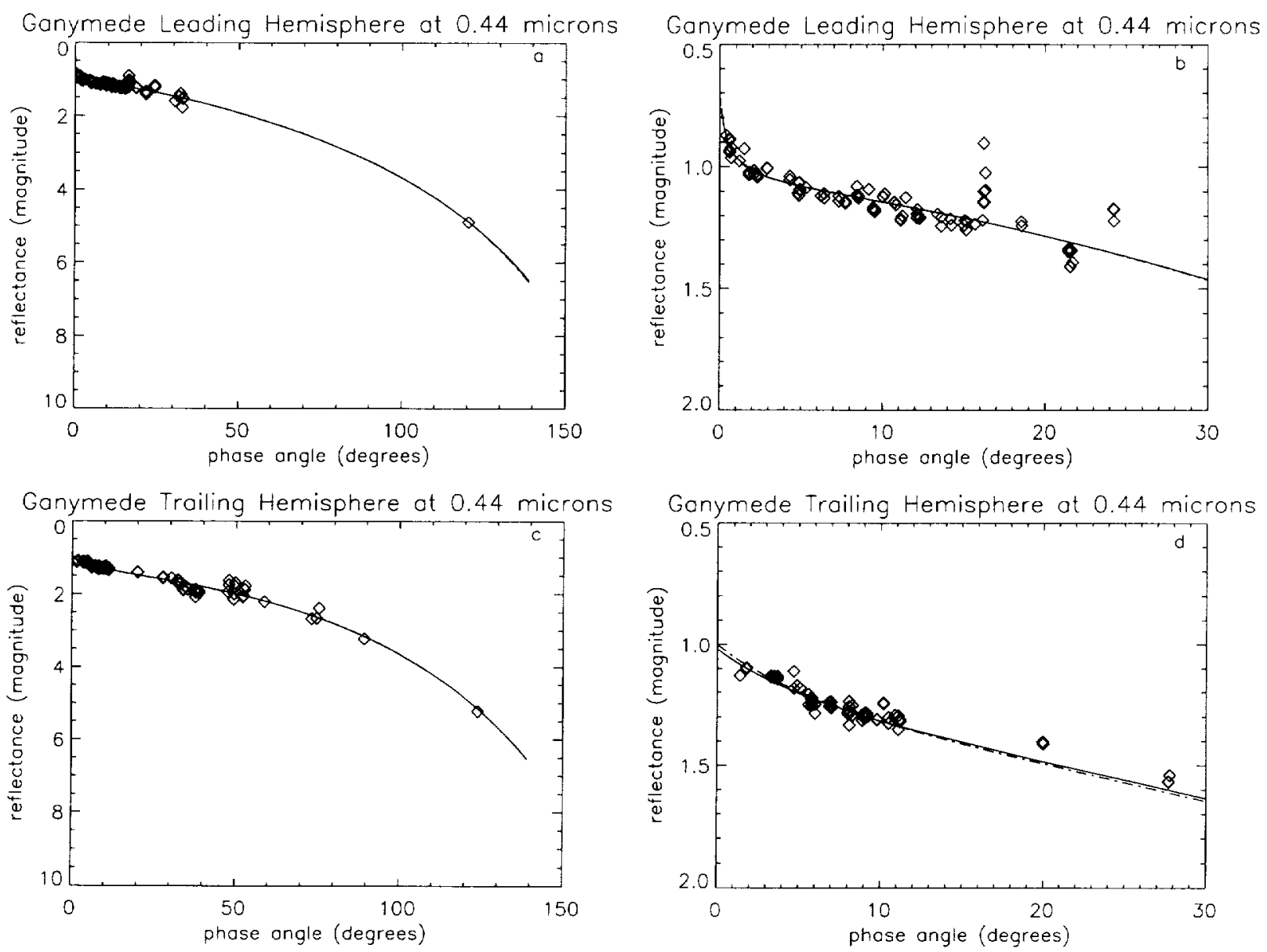

FIG. 4. The solar phase curves of Ganymede's leading ( $a$ and $b$ ) and trailing ( $c$ and $d$ ) hemispheres at $0.47 \mu \mathrm{m}$, where the diamonds represent the disk-integrated observations, the solid line represents the isotropic multiple scattering Hapke model solution incorporating a two parameter HG function, the dashed line represents the isotropic multiple scattering Hapke model solution incorporating a three parameter HG function, and the dash-dot line represents the anisotropic multiple scattering Hapke model solution incorporating a three parameter HG function. Those places where only a solid line is seen indicates that the solutions lie on top of each other and, therefore, cannot be distinguished.

The opposition parameter $h$ is dependent on the grain size distribution and porosity within the optically active portion of the regolith. These physical characteristics are also independent of the wavelength of observation. Analysis of the phase curves of the same surface at different wavelengths in addition to good quality disk-integrated measurements at very low phase angles $\left(\alpha<1^{\circ}\right)$ constrains the values of this parameter to wihin \pm 0.0005 for all the icy satellite data except for Ganymede's trailing hemisphere which lacks the very low phase angle data. The value of the opposition width parameter, $h$, is strongly coupled to the value of bo, the opposition amplitude. Using the wavelength independent nature of $h$ and the quality of the very low phase angle data, the value of bo can be constrained to \pm 0.02 for all the data sets excluding Ganymede's trailing hemisphere.

Additional constraints on the error bars for the Hapke parameters may also be based on the method of analysis. For the data sets fits using a modified least squares grid search, differences in RMS values of $1 \%$ are considered significant and error bars can be established on this basis. The modified least squares grid search is an iterative process where the initial grid size is coarse and the solutions with the 10 least RMS values are all considered viable solutions to the model. A second grid search is run where the limits of the grid for a specific parameter are based on the range of values found from the first 10 solutions plus and minus the initial grid increment and the grid size is reduced. Once again the solutions with the 10 least RMS values are considered viable solutions to the model and the process is repeated until either the desired grid size is reached or the differences in the least 10 RMS values is less than $1 \%$. For example, the parameter $w$ will initially be run from values of 0.2 to 1.0 in increments of 0.2 . The 

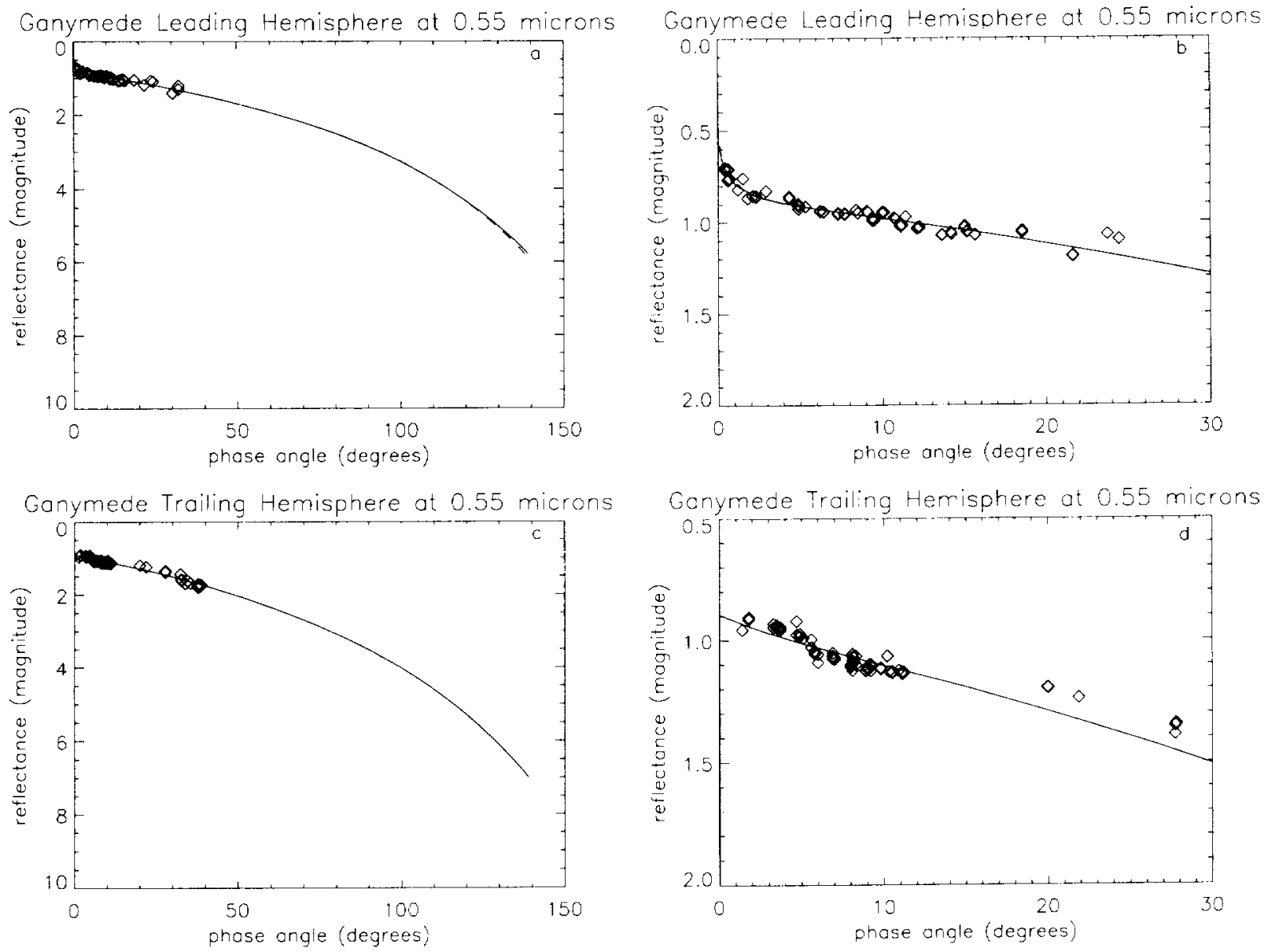

FIG. 5. The solar phase curves of Ganymede's leading (a and b) and trailing (c and d) hemispheres at $0.55 \mu \mathrm{m}$, where the diamonds represent the disk-integrated observations, the solid line represents the isotropic multiple scattering Hapke model solution incorporating a two parameter HG function, and the dashed line represents the isotropic multiple scattering Hapke model solution incorporating a three parameter HG function. Those places where only a solid line is seen indicates that the solution lie on top of each other, and therefore. cannot be distinguished.

range of values for $w$ from the first set of 10 lesat RMS solutions may range from 0.6 to 0.8 . Then the next grid search run will have values for $w$ ranging from 0.4 to 1.0 in increments of 0.1 . All the parameters are treated in the same manner. The error bars listed in Table II correspond to the smallest grid increment run on that parameter. However, by examining the parameter values for the 10 least RMS solutions along each step in the grid search, coupled with the error analysis results discussed above, the following final error bars can be set for the Hapke parameter values (in the isotropic multiple scattering case) for the icy Galilean satellites: $w= \pm 0.01$ for all the satellites, bo $= \pm 0.02$ for all the satellites except Ganymede's trailing hemisphere where the value is \pm 0.1 , $h= \pm 0.0005$ for all the satellites except Ganymede's trailing hemisphere where the value is $\pm 0.01, \theta$-bar $= \pm 5^{\circ}$ except for Ganymede's $0.55-\mu \mathrm{m}$ phase curves where the error bars are $\pm 10^{\circ}$ and Callisto's leading hemisphere phase curves where the $\theta$-bar error bars are $\pm 15^{\circ}$. The final error bars for the scattering function parameters are $b_{2}=c_{2}=c_{3}= \pm 0.02$ and $d 3= \pm 0.01$. The error bars for $b_{3}$ are \pm 0.05 for Europa's 0.47 - $\mu \mathrm{m}$ phase curves, Europa's $0.55-\mu \mathrm{m}$ leading hemisphere phase curve, Ganymede's $0.47-\mu \mathrm{m}$ phase curves, and Callisto's trailing hemisphere phase curves. For Europa's $0.55-\mu \mathrm{m}$ trailing hemisphere phase curve the $b_{3}$ error bar is \pm 0.1 and is \pm 0.5 for Ganymede's $0.55-\mu \mathrm{m}$ phase curves and Callisto's leading hemisphere phase curves.

The phase curve analyses incorporating anisotropic multiple scattering were conducted using software designed by Paul Helfenstein following the method outlined by Verbiscer (1991). The error analysis in the software utilized a gradient-expansion search of the $\chi^{2}$ parameter space which locates the minimum between measured and calculated reflectance values. Details of this method are given in $\mathrm{Be}-$ vington (1969) and Helfenstein (1986). 

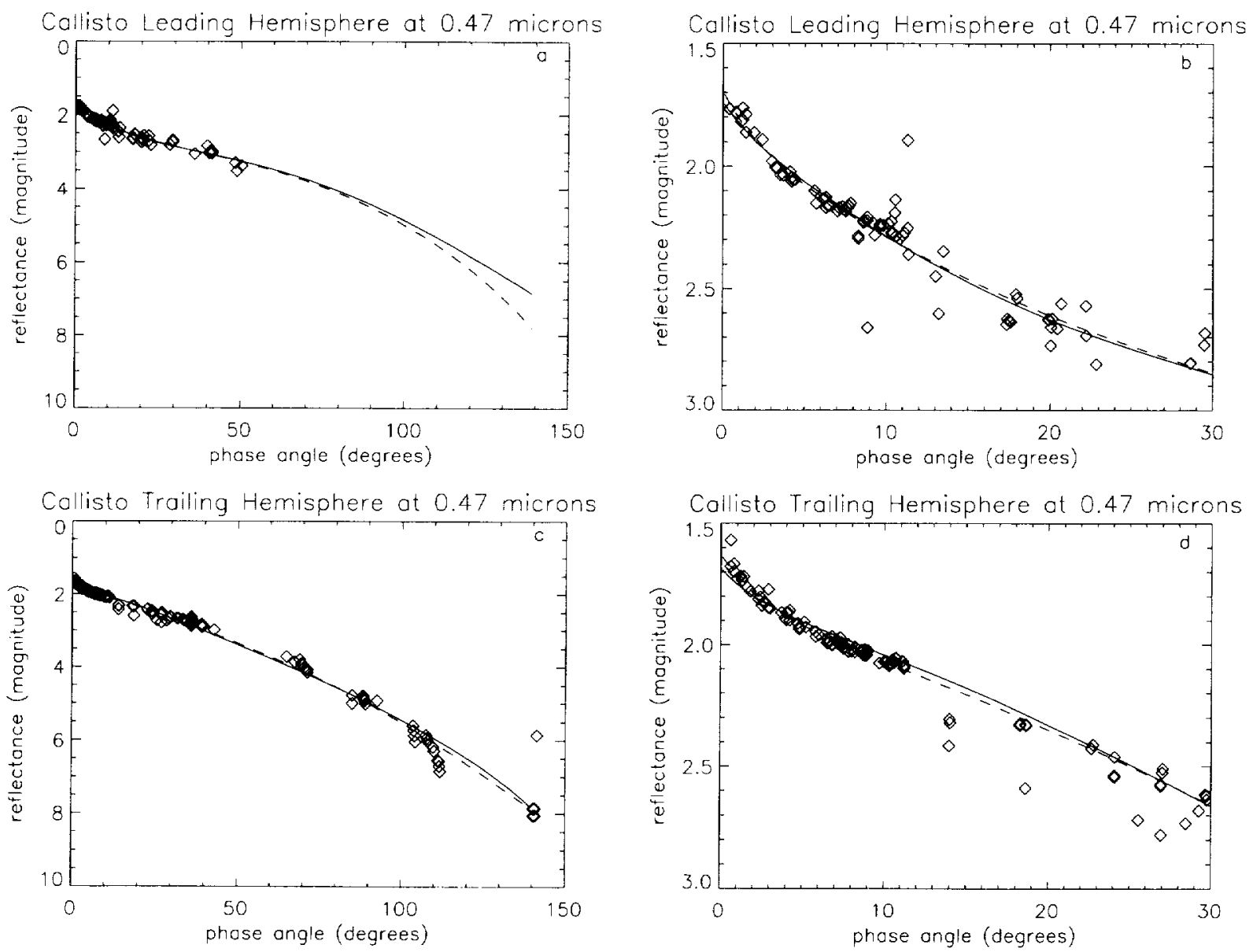

FIG. 6. The solar phase curves of Callisto's leading ( $a$ and b) and trailing ( $c$ and d) hemispheres at $0.47 \mu$ m, where the diamonds represent the disk-integrated observations, the solid line represents the isotropic multiple scattering Hapke model solution incorporating a two parameter HG function, the dashed line represents the isotropic multiple scattering Hapke model solution incorporating a three parameter HG function. Those places where only a solid line is seen indicates that the solutions lie on top of each other and, therefore, cannot be distinguished.

\section{RESULTS}

\section{Isotropic Multiple Scattering Results}

General. Hapke's theory models the phase curves of these satellites very well, as demonstrated in Figs. 2 through 7. There is no substantial difference in the quality of fit between the model incorporating the 3P-HG function over the model incorporating the $2 \mathrm{P}-\mathrm{HG}$ function. The largest difference in RMS values is less than $2 \%$, where as the majority of fits are within $0.1 \%$. There were only four phase curves for which no large phase angle $\left(\alpha>60^{\circ}\right)$ data were available. In the case of the $0.55-\mu \mathrm{m}$ data for both hemispheres of Ganymede the two models predict the same behavior at large phase angles. However, for the leading hemisphere phase curves of Callisto the two models predictions at large phase angles diverge significantly. Therefore the results of the models for these phase curves must be interpreted with caution.
Figures 8 through 10 are comparisons of both model predictions versus disk-resolved scans across the satellite surfaces. Each scan is a vertical scan from north to south across an orthographic projection of the image where the values of incidence, emission, latitude, and longitude angles and pixel brightness are recorded based on the image label information. Figure 8 is a scan across Europa where both models fit the lower albedo data (which corresponds to mottled terrains), but do not model the bright plains units at low and high pixel locations. This type of fit is to be expected when albedo varies across the surface. Figure 9 is a scan across Ganymede where the two models made very different predictions of the surface brightness as a function of location, but each fits the data equally well. The 2P-HG model fits the higher albedo regions, whereas the $3 \mathrm{P}-\mathrm{HG}$ model fits the lower albedo regions. Once again, these types of fits are to be expected when albedo varies across the surface. Figure 10 is a scan across 

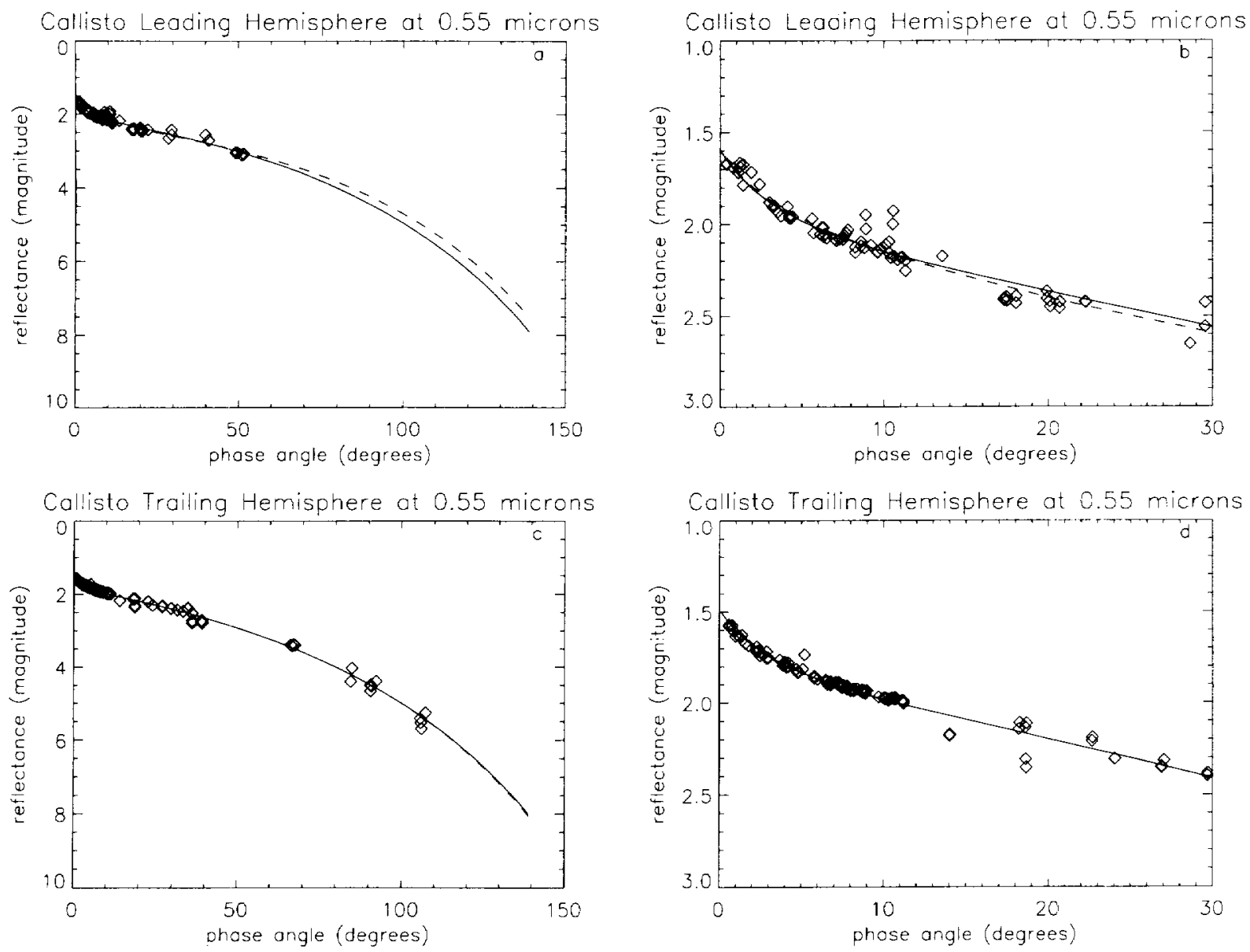

FIG. 7. The solar phase curves of Callisto's leading ( $\mathrm{a}$ and $\mathrm{b}$ ) and trailing (c and d) hemispheres at $0.55 \mu \mathrm{m}$, where the diamonds represent the disk-integrated observations, the solid line represents the isotropic multiple scattering Hapke model solution incorporating a two parameter HG function, the dashed line represents the isotropic multiple scattering Hapke model solution incorporating a three parameter HG function. Those places where only a solid line is seen indicates that the solutions lie on top of each other and, therefore, cannot be distinguished.

the surface of Callisto where both models fit the data equally well.

Roughness. The macroscopic roughness parameter, $\theta$ bar, shows no hemispherical differences on Europa or Callisto in both models. This agrees with the results Domingue et al. (1991) obtained for Europa, but contradicts the results Buratti (1991) obtained for Callisto. Buratti (1991) found that the leading hemisphere of Callisto is macroscopically rougher than its trailing hemisphere. The discrepancy between our results and those of Buratti (1991) is due to the fact that neither study had large phase angle data for Callisto's leading hemisphere. In Hapke's model it is essential to have large phase angle data in order to constrain the macroscopic roughness parameter. Since there are currently no large phase angle data for Callisto's leading hemisphere, it cannot be stated with certainty if there is a hemispherical difference in surface roughness on Callisto. The shape of the single scattering function is also greatly influ- enced by large phase angle observations. Therefore the different choices in single scattering function coupled with the lack of large phase angle data also contribute to the different roughness results between the two studies. No differences in the value of roughness were seen for Callisto between the 2P-HG model and the 3P-HG model. The values of $\theta$-bar for Europa between the two models are within $1^{\circ}$ of each other, well within the error bars. Both models show that the trailing hemisphere of Ganymede is rougher than the leading hemisphere by about $6^{\circ}$. Buratti (1991) found no hemispherical differences in roughness in her analysis of Ganymede, which we attribute to the choice of single particle scattering function. Hartman and Domingue (1997) found that the two models returned very different values of $\theta$-bar when applied to the lunar phase curve, up to $12^{\circ}$ difference. However, no differences within each satellite were found outside the error bars. An increasing trend in the $\theta$-bar values with distance from Jupi- 
TABLE III

Anisotropic Multiple Scattering Hapke Model Results

\begin{tabular}{|c|c|c|c|c|c|c|c|c|}
\hline & \multicolumn{4}{|c|}{ Leading } & \multicolumn{4}{|c|}{ Trailing } \\
\hline & $0.47 \mu$ & Error bar & $0.55 \mu \mathrm{m}$ & Error bar & $0.47 \mu \mathrm{m}$ & Error bar & $0.55 \mu \mathrm{m}$ & Error bar \\
\hline $\mathrm{w}$ & 0.905 & \pm 0.001 & 0.949 & \pm 0.002 & 0.897 & \pm 0.024 & 0.911 & \pm 0.002 \\
\hline bo & 0.45 & \pm 0.19 & 0.5 & \pm 0.17 & 0.74 & \pm 0.19 & 0.301 & \pm 0.041 \\
\hline $\mathrm{h}$ & 0.0019 & \pm 0.006 & 0.0013 & \pm 0.0029 & 0.0009 & \pm 0.0015 & 0.012 & \pm 0.005 \\
\hline b3 & 0.5 & $n c$ & 0.0 & \pm 0.1 & 0.0 & nc & 0.0003 & \pm 0.01 \\
\hline $\mathrm{c} 3$ & 0.972 & \pm 0.017 & 0.967 & \pm 0.04 & 0.681 & \pm 0.102 & 0.988 & \pm 0.007 \\
\hline d3 & -0.43 & \pm 0.01 & -0.4185 & \pm 0.01 & -0.418 & \pm 0.107 & -0.333 & \pm 0.001 \\
\hline$\theta$-bar & 2. & \pm 5 & 0.1 & \pm 5 & 13.7 & \pm 2.6 & 0 & \pm 5 \\
\hline $\mathrm{rms}$ & 0.027 & & 0.03 & & 0.035 & & 0.023 & \\
\hline$w$ & 0.8227 & \pm 0.02 & $\mathrm{n} / \mathrm{a}$ & $\begin{array}{l}\text { Ganymede } \\
\text { n/a }\end{array}$ & 0.873 & \pm 0.016 & $\mathrm{n} / \mathrm{a}$ & $\mathrm{n} / \mathrm{a}$ \\
\hline bo & 0.5537 & \pm 0.1762 & $\mathrm{n} / \mathrm{a}$ & $\mathrm{n} / \mathrm{a}$ & 1.2154 & \pm 0.2722 & $\mathrm{n} / \mathrm{a}$ & $\mathrm{n} / \mathrm{a}$ \\
\hline $\mathrm{h}$ & 0.0035 & \pm 0.0045 & $\mathrm{n} / \mathrm{a}$ & $\mathrm{n} / \mathrm{a}$ & 0.0758 & \pm 0.016 & $\mathrm{n} / \mathrm{a}$ & $\mathrm{n} / \mathrm{a}$ \\
\hline b3 & 0.01 & nc & $\mathrm{n} / \mathrm{a}$ & $\mathrm{n} / \mathrm{a}$ & 0.0395 & nc & $\mathrm{n} / \mathrm{a}$ & $\mathrm{n} / \mathrm{a}$ \\
\hline c3 & -0.92 & \pm 0.1 & $\mathrm{n} / \mathrm{a}$ & $\mathrm{n} / \mathrm{a}$ & 0.5592 & \pm 0.12 & $\mathrm{n} / \mathrm{a}$ & $\mathrm{n} / \mathrm{a}$ \\
\hline d3 & -0.29 & \pm 0.09 & $\mathrm{n} / \mathrm{a}$ & $\mathrm{n} / \mathrm{a}$ & -0.0422 & \pm 0.05 & $\mathrm{n} / \mathrm{a}$ & $\mathrm{n} / \mathrm{a}$ \\
\hline $\begin{array}{l}\theta \text {-bar } \\
\text { rms }\end{array}$ & $\begin{array}{l}27.4 \\
0.067\end{array}$ & \pm 2.6 & $\mathrm{n} / \mathrm{a}$ & $\mathrm{n} / \mathrm{a}$ & $\begin{array}{c}35.06 \\
0.096\end{array}$ & \pm 1.6 & $\mathrm{n} / \mathrm{a}$ & $\mathrm{n} / \mathrm{a}$ \\
\hline
\end{tabular}

Note. nc, not calculated, na, not available.

ter is seen, which correlates with the amount of large impact cratering observed on these satellites and therefore the age of their surfaces.

Opposition effect. The opposition width parameter, $h$, showed no differences outside the error bars between the $2 \mathrm{P}-\mathrm{HG}$ model and the $3 \mathrm{P}-\mathrm{HG}$ model, consistent with the findings of Hartman and Domigue (1997). No hemispherical differences were seen on Europa; however, both Ganymede and Callisto displayed variations with hemisphere. The value of the opposition width parameter is dependent on the porosity and grain size distribution of the particles within the optically active portion of the regolith. Generally the larger the value of this parameter the more compact or less porous the regolith. The correlation between $h$ and grain size is less straightforward. Therefore the values of $h$ seen for both hemispheres of Europa indicate a uniform, highly porous, surface. The results shown in Table II indicate that the leading hemisphere of Ganymede is much more porous than the trailing hemisphere. The surprising result is that the value of $h$ for the trailing hemisphere of Ganymede is even larger than that obtained by Hartman and Domingue (1997) for the Moon! Buratti (1991) found no hemispherical differences in the compaction state of Ganymede's regolith. The results for Callisto show that its leading hemisphere is slightly more compact than its trailing hemisphere. Buratti (1991) found the opposite; how-

TABLE IV

Isotropic Model Error Bars

\begin{tabular}{|c|c|c|c|c|c|c|c|c|c|c|}
\hline Satellite & Hemisphere & $w$ & bo & $\mathrm{h}$ & $\mathrm{b}_{2}$ & $c_{2}$ & $\mathrm{~b}_{3}$ & $\mathrm{~d}_{3}$ & $c_{3}$ & $\theta$-bar \\
\hline \multirow[t]{4}{*}{ Europa } & (0.47- $\mu \mathrm{m}$ lead & 0.01 & 0.02 & 0.0005 & 0.02 & 0.02 & 0.05 & 0.01 & 0.02 & 5 \\
\hline & $0.47-\mu \mathrm{m}$ trail & 0.01 & 0.02 & 0.0005 & 0.02 & 0.02 & 0.05 & 0.01 & 0.02 & 5 \\
\hline & $0.55-\mu \mathrm{m}$ lead & 0.01 & 0.02 & 0.0005 & 0.02 & 0.02 & 0.05 & 0.01 & 0.02 & 5 \\
\hline & $0.55 \mu \mathrm{m}$ trail & 0.01 & 0.02 & 0.00015 & 0.02 & 0.02 & 0.1 & 0.01 & 0.02 & 5 \\
\hline \multirow[t]{4}{*}{ Ganymede } & $0.47-\mu \mathrm{m}$ lead & 0.01 & 0.02 & 0.0005 & 0.02 & 0.02 & 0.05 & 0.01 & 0.02 & 5 \\
\hline & $0.47-\mu \mathrm{m}$ trail & 0.01 & 0.1 & 0.01 & 0.02 & 0.02 & 0.05 & 0.01 & 0.02 & 5 \\
\hline & $0.55-\mu \mathrm{m}$ lead & 0.01 & 0.02 & 0.0005 & 0.02 & 0.02 & 0.5 & 0.01 & 0.02 & 10 \\
\hline & $0.55 \mu \mathrm{m}$ trail & 0.01 & 0.1 & 0.01 & 0.02 & 0.02 & 0.5 & 0.01 & 0.02 & 10 \\
\hline \multirow[t]{4}{*}{ Callisto } & $0.47-\mu \mathrm{m}$ lead & 0.01 & 0.02 & 0.0005 & 0.02 & 0.02 & 0.5 & 0.01 & 0.02 & 15 \\
\hline & $0.47-\mu \mathrm{m}$ trail & 0.01 & 0.02 & 0.0005 & 0.02 & 0.02 & 0.05 & 0.01 & 0.02 & 5 \\
\hline & $0.55-\mu \mathrm{m}$ lead & 0.01 & 0.02 & 0.0005 & 0.02 & 0.02 & 0.5 & 0.01 & 0.02 & 15 \\
\hline & $0.55 \mu \mathrm{m}$ trail & 0.01 & 0.02 & 0.0005 & 0.02 & 0.02 & 0.05 & 0.01 & 0.02 & 5 \\
\hline
\end{tabular}




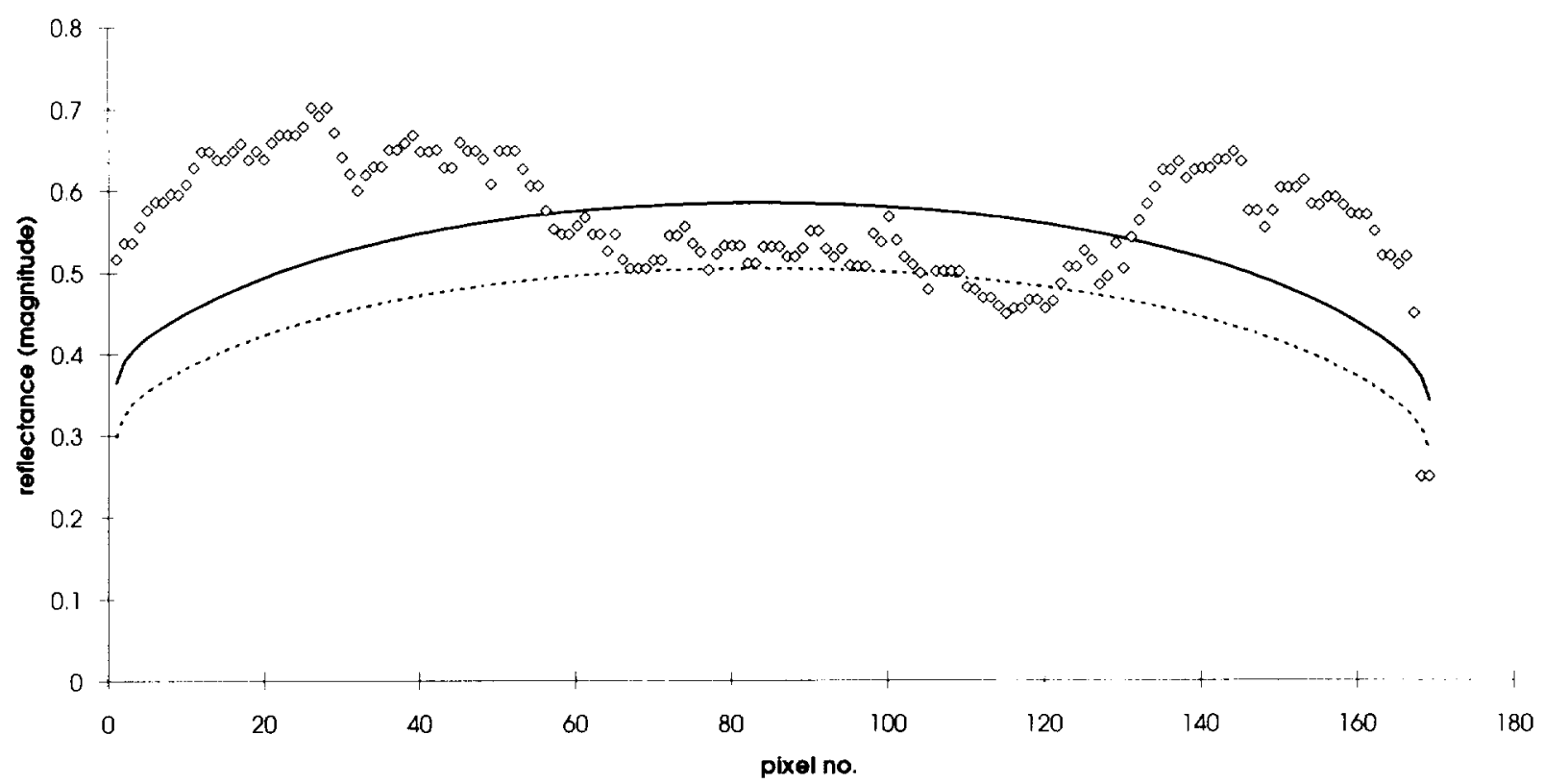

FIG. 8. Vertical scan from north to south across an orthographic projection of the Voyager image 16342.22 of Europa. This image is an orange filter image of the trailing hemisphere. The diamonds represent the Voyager data, the solid line represents the isotropic multiple scattering $2 \mathrm{P}$-HG Hapke model solution, and the dashed line represents the isotropic multiple scatteing 3P-HG Hapke model solution.

ever, our phase curve contains opposition data which was not available at the time she performed her study. The differences in the results obtained by Buratti (1991) and this study for Ganymede are not understood and may be attributed to the lack of the very low phase angle data needed to well define the opposition effect.

There are variations in the value of the opposition amplitude parameter, bo, between the $2 \mathrm{P}-\mathrm{HG}$ function and the

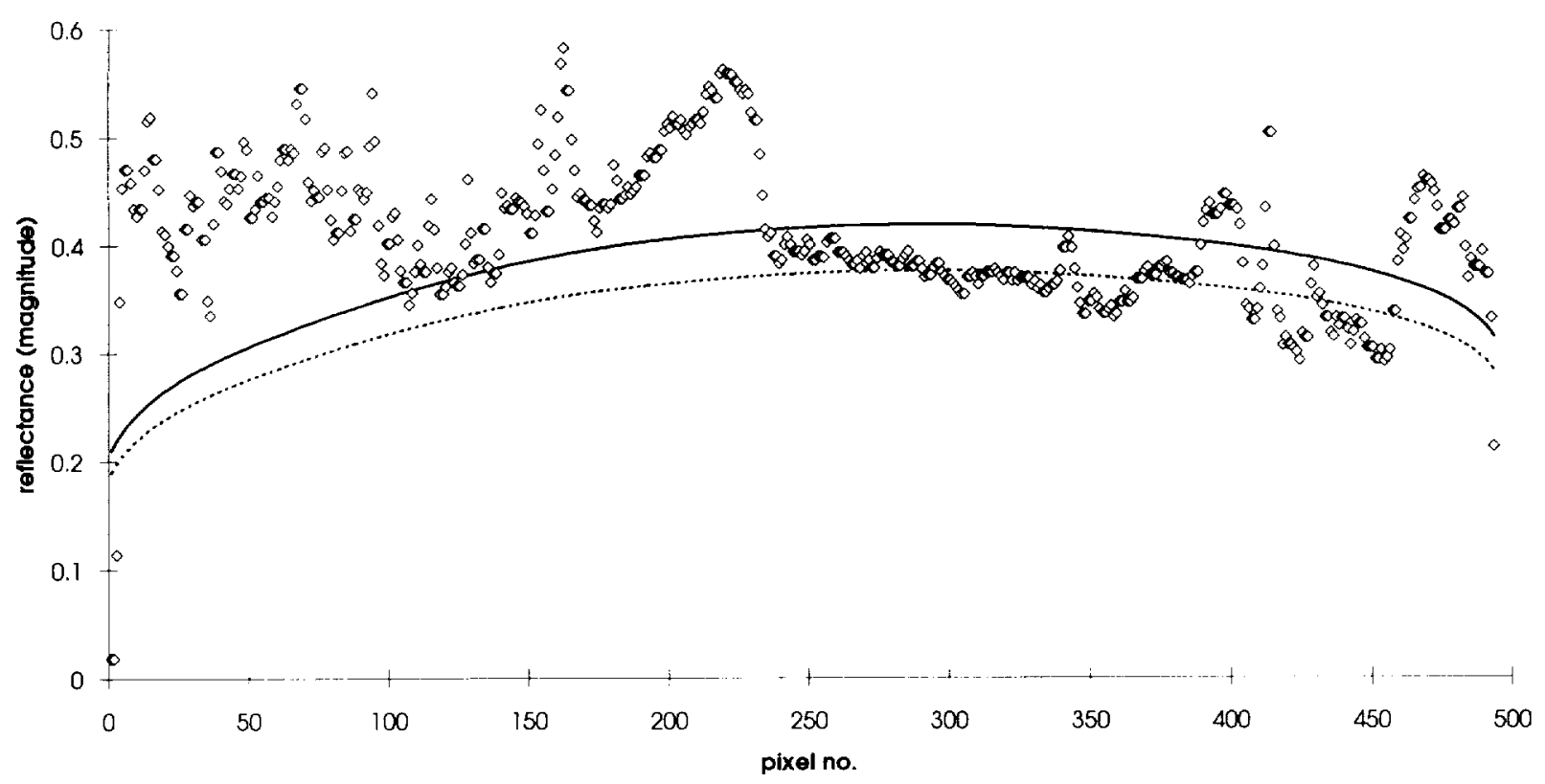

FIG. 9. Vertical scan from north to south across an orthographic projection of the Voyager image 20635.35 of Granymede. This image is an orange filter image of the trailing hemisphere. The diamonds represent the Voyager data, the solid line represents the isotropic multiple scattering 2P-HG Hapke model solution, and the dashed line represents the isotropic multiple scattering 3P-HG Hapke model solution. 


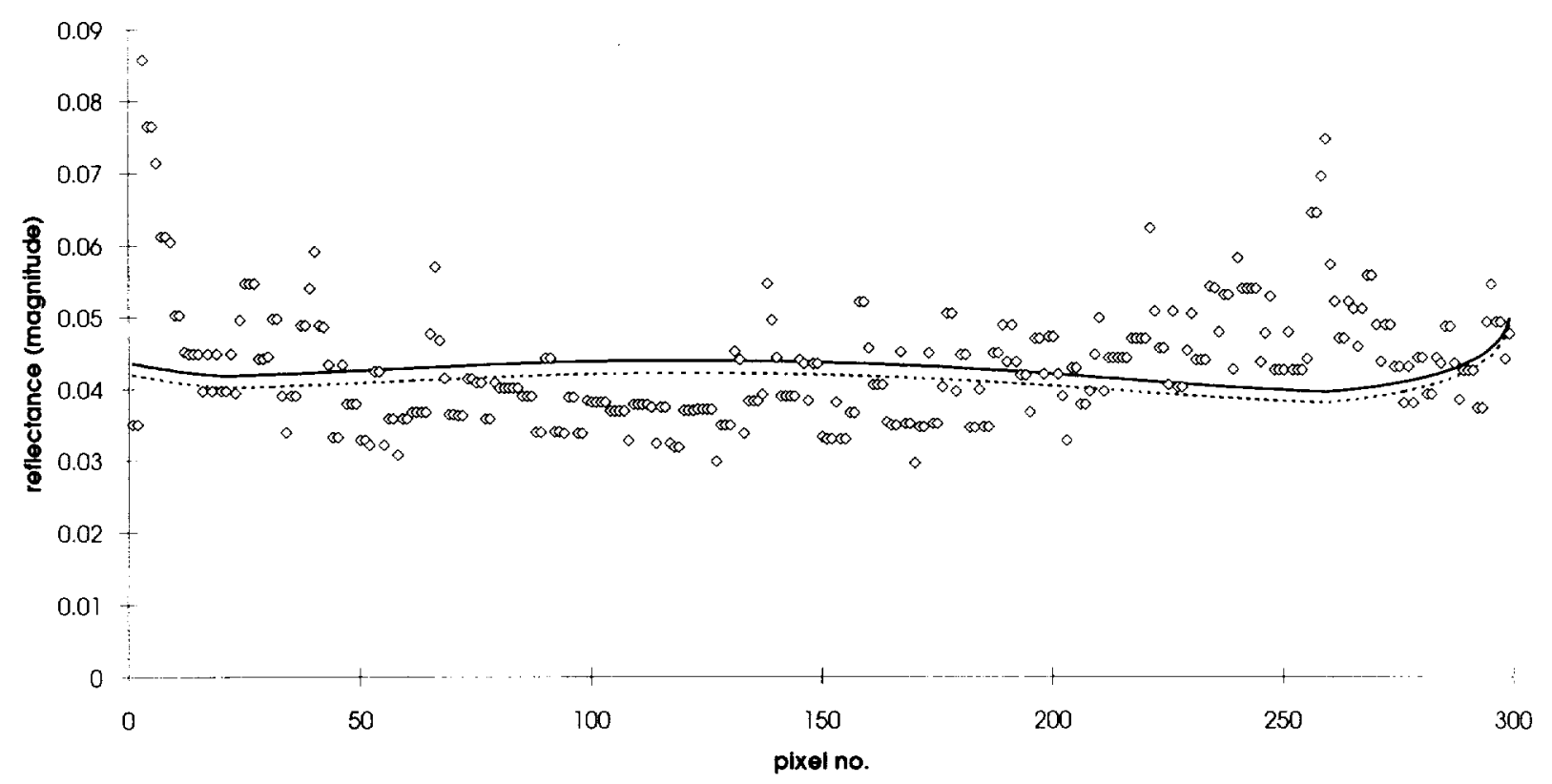

FIG. 10. Vertical scan from north to south across an orthographic projection of the Voyager image 20616.11 of Callisto. This image is an orange filter image of the trailing hemisphere. The diamonds represent the Voyager data, the solid line represents the isotropic multiple scattering $2 \mathrm{P}-\mathrm{HG}$ Hapke model solution, and the dashed line represents the isotropic multiple scattering 3P-HG Hapke model solution.

3P-HG function, which was not found by Hartman and Domingue (1997) in their similar analysis of the Moon. In some cases the difference is substantial, in others the differences are within the error bars of the parameters. The opposition parameter bo is correlated to the opacity of the regolith particles. It is a measure of the percentage of incident light which is scattered at the surface of the particle compared to the total amount of light scattered (both from the surface and internally). A bo value of one indicates an opaque particle where all the light is scattered at the particle surface. In the case of Europa both models return values of bo near one-half for both hemispheres, indicating a fairly transparent material, which is consistent with a predominately water ice surface. The results for Ganymede show variations between hemispheres and between wavelengths. Both models indicate that on the leading hemisphere the material is more opaque at $0.55 \mu \mathrm{m}$ than at $0.44 \mu \mathrm{m}$, while the opposite is true on the trailing hemisphere. The trailing hemisphere values of bo do not vary between the two models; however, there is a significant difference in the bo values returned by the models for the leading hemisphere at $0.55 \mu \mathrm{m}$. The $3 \mathrm{P}-\mathrm{HG}$ model indicates a more opaque material than the $2 \mathrm{P}-\mathrm{HG}$ model. The results for Callisto also demonstrate variations between hemispheres and wavelengths. Both models show that the material on the leading hemisphere is more opaque than the material on the trailing hemisphere; however, the 2P-HG model indicates larger differences in opacity at 0.47 $\mu \mathrm{m}$ than the $3 \mathrm{P}-\mathrm{HG}$ model. The $2 \mathrm{P}-\mathrm{HG}$ model indicates significantly more opaque material at $0.47 \mu \mathrm{m}$ on the leading hemisphere than the $3 \mathrm{P}-\mathrm{HG}$ model, while it indicates significantly more transparent material at both wavelengths on the trailing hemisphere than the $3 \mathrm{P}-\mathrm{HG}$ model.

Single scattering parameters. Hapke's theory also returns a measure of the single scattering albedo, $w$. The values of $w$ vary significantly between the two model results. The $2 \mathrm{P}-\mathrm{HG}$ model finds a lower $w$ value for Europa's leading hemisphere at $0.47 \mu \mathrm{m}$, but returns a similar value as the 3P-HG model for Europa's leading hemisphere at $0.55 \mu \mathrm{m}$. The $2 \mathrm{P}-\mathrm{HG}$ model also finds similar values as the 3P-HG model for Europa's trailing hemisphere at 0.47 $\mu \mathrm{m}$, but returns a brighter value for this hemisphere at $0.55 \mu \mathrm{m}$. The only differences in $w$ found by these two models for the hemispheres of Ganymede is for the leading hemisphere at $0.55 \mu \mathrm{m}$, in which the $2 \mathrm{P}-\mathrm{HG}$ model finds a brighter value than the $3 \mathrm{P}-\mathrm{HG}$ model. The differences for Callisto are very large. The 3P-HG model finds significantly brighter $w$ values at both wavelengths for the trailing hemisphere and at $0.55 \mu \mathrm{m}$ for the leading hemisphere. However the 3P-HG model finds a darker value for the leading hemisphere at $0.47 \mu \mathrm{m}$ than the $2 \mathrm{P}-\mathrm{HG}$ model.

Figures 11 through 13 plot and compare the single scattering functions from the models for each satellite. Both isotropic multiple scattering models show that Europa's leading hemisphere is backscattering at both wavelengths; however, the isotropic 3P-HG model also predicts a significant forward scattered component. In the case of Euro- 

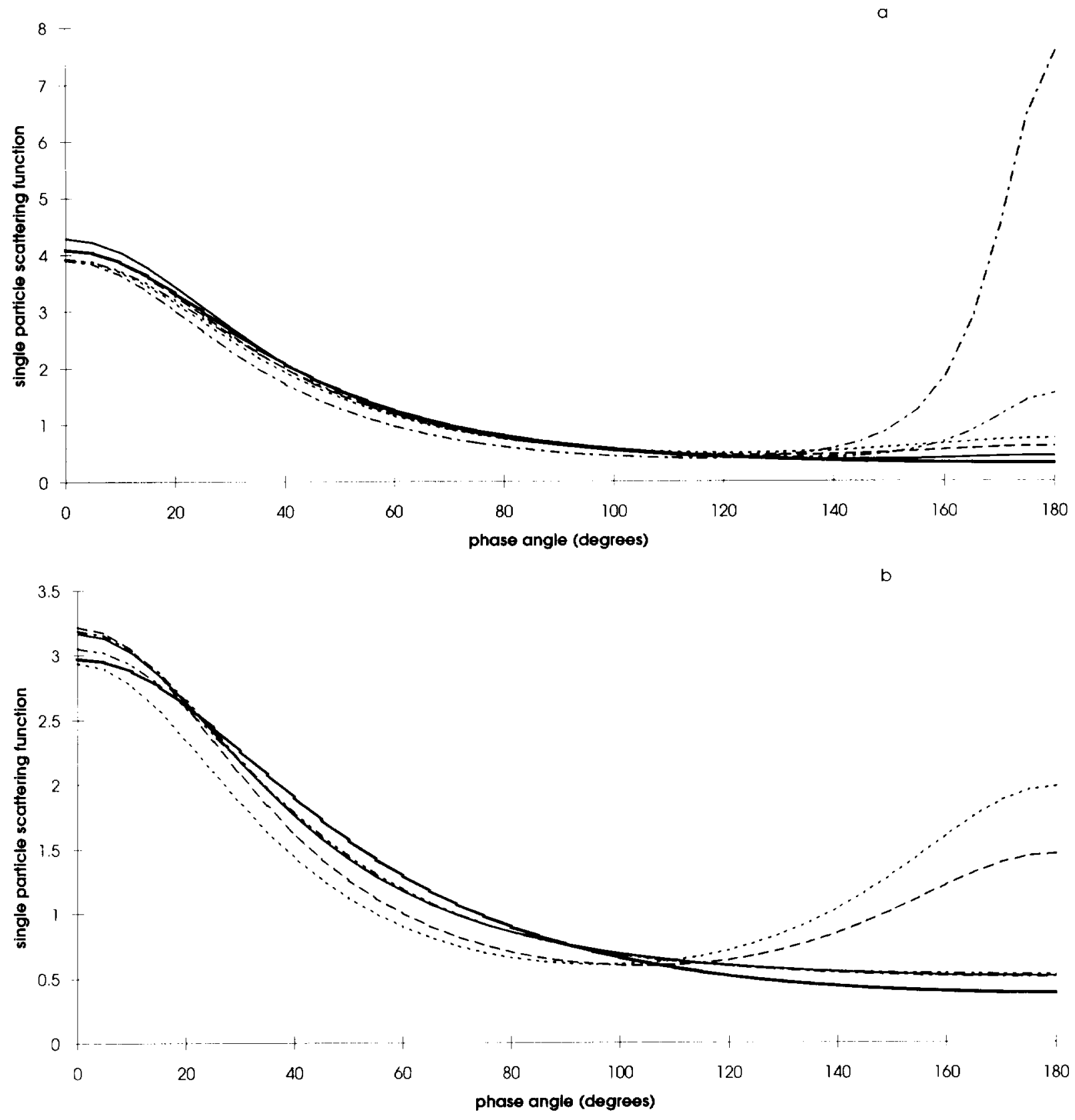

FIG. 11. The single particle scattering functions for Europa's (a) leading and (b) trailing hemispheres plotted versus phase angle. The long and shorl dashed lines represent the isotropic multiple scaltering model with the two parameter HG function solutions at 0.47 and $0.55 \mu \mathrm{m}$. respectively. The dash-dot and dash-double-dot lines represent the isotropic multiple scattering model with the three parameter HG function solutions at 0.47 and $0.55 \mu \mathrm{m}$. respectively. The thin and thick solid lines represent the anisotropic multiple scattering model with the three parameter HG function solutions at 0.47 and $0.55 \mu \mathrm{m}$, respectively. Those areas where only one solution is apparent indicate areas where the scattering functions lie on top of each other and, therefore, cannot be disginguished.

pa's trailing hemisphere the isotropic $2 \mathrm{P}-\mathrm{HG}$ function shows a significant forward scattering lobe. The isotropic 3P-HG function, on the other hand, shows no evidence of forward scattering on Europa's trailing hemisphere in either wavelength. Both isotropic scattering models show that both hemispheres of Europa have a significant back- scattering component, with the amplitude of the backscattering lobe stronge on the leading hemisphere.

Figure 12 shows the single particle scattering behavior for Ganymede. Both isotropic 2P-HG and 3P-HG functions show some interesting hemispherical and wavelength dependent differences. At $0.47 \mu \mathrm{m}$ the single scattering 

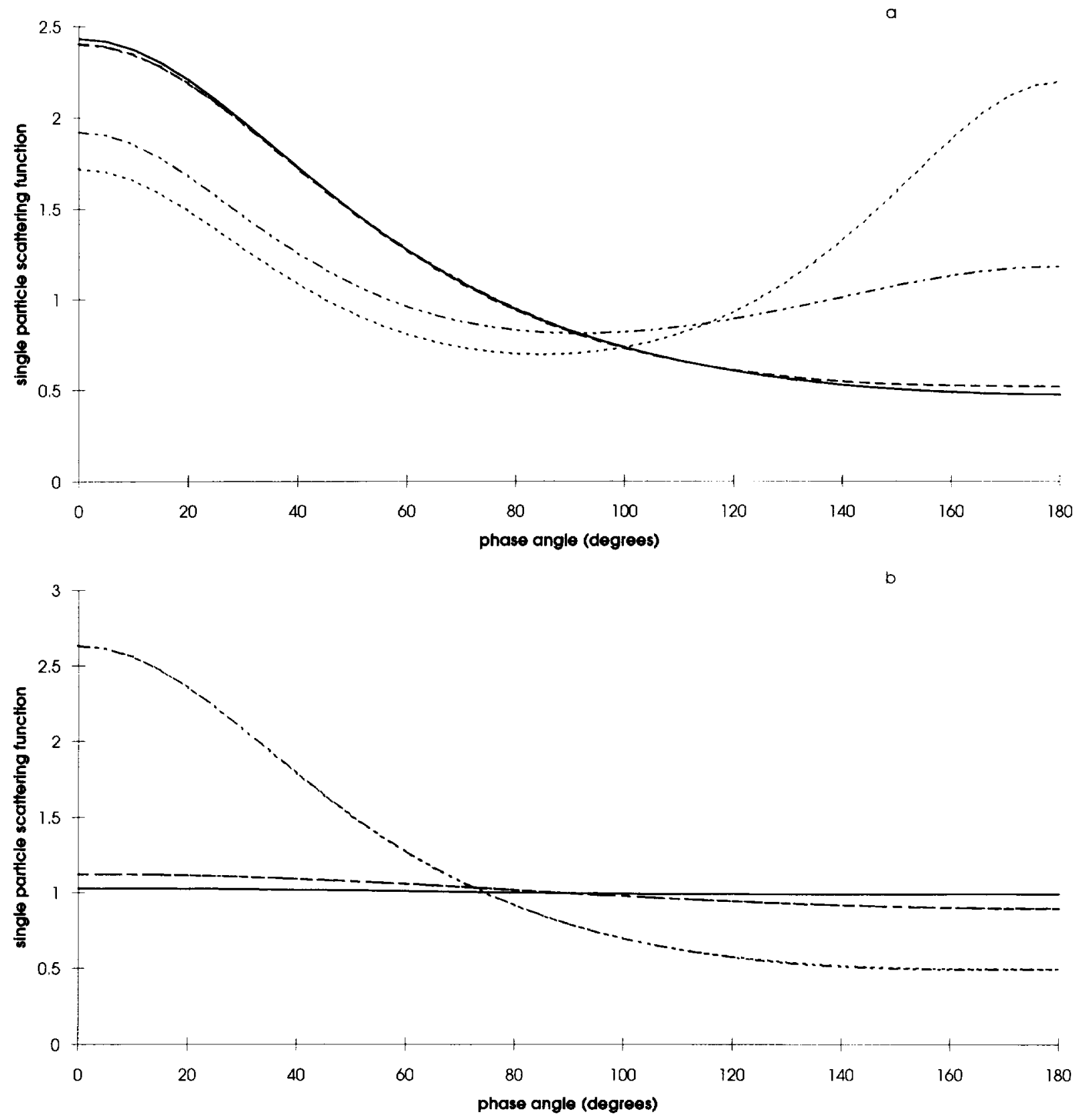

FIG. 12. The single particle scattering functions for Ganymede's (a) leading and (b) trailing hemispheres plotted versus phase angle. The long and short dashed lines represent the isotropic multiple scattering model with the two parameter HG function solutions at 0.47 and $0.55 \mu \mathrm{m}$, respectively. The dash-dot and dash-double-dot lines represent the isotropic multiple scattering model with the three parameter HG function solutions at 0.47 and $0.55 \mu \mathrm{m}$, respectively. The solid lines represent the anisotropic multiple scattering model with the three parameter HG function solutions at $0.47 \mu \mathrm{m}$. Those areas where only one solution is apparent indicate areas where the scattering functions lie on top of each other and, therefore, cannot be distinguished.

function predicted by both models is isotropic on the trailing side, while the leading hemisphere it is backscattering. At $0.55 \mu \mathrm{m}$ on the trailing hemisphere both isotropic multiple scattering models show a backscattering scattering function; however, for the leading hemisphere the isotropic $2 \mathrm{P}-\mathrm{HG}$ model predicts a slightly stronger forward scatter- ing component. The isotropic 3P-HG function at $0.55 \mu \mathrm{m}$ predicts that both hemispheres are predominately backscattering with little difference between the two. However, there was no large phase angle data for the leading hemisphere of Ganymede at $0.55 \mu \mathrm{m}$, so the results for the leading hemisphere are preliminary. These findings dis- 

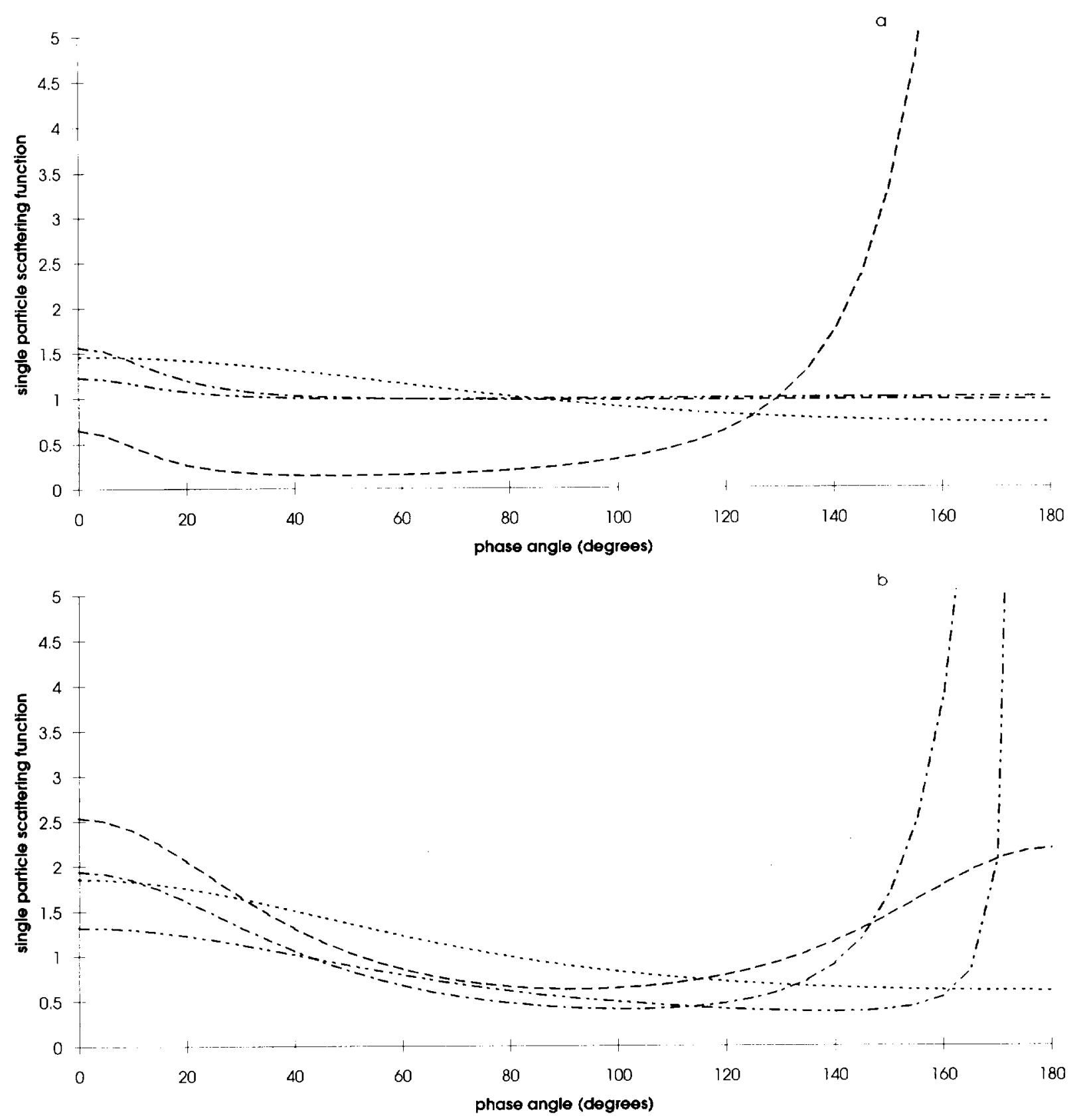

FIG. 13. The single particle scattering functions for Callisto's (a) leading and (b) trailing hemispheres plotted versus phase angle. The solid and dashed lines represent the isotropic multiple scattering model with the two parameter HG function solutions at 0.47 and $0.55 \mu \mathrm{m}$. respectively. The dash-double-dot and dash-dot lines represent the isotropic multiple scattering model with the three parameter HG function solutions at 0.47 and $0.55 \mu \mathrm{m}$, respectively. Those areas where only one solution is apparent indicates areas where the scattering functions lie on top of each other and, therefore, cannot be distinguished.

agree with Buratti’s (1991) results that there are no hemispherical differnces in the single scattering behavior on Ganymede; however, this may be an artifact of the different single particle scattering functions chosen between the two studies.

Figure 13 shows the single particle scattering behavior for Callisto. Recall that there were no large phase angle data for the leading hemisphere at either wavelength, so the leading hemisphere results need to be interpreted with great caution. The isotropic multiple scattering 3P-HG function predicts that the leading hemisphere's particles are nearly isotropic, whereas the trailing hemisphere is more backscattering with some forward scattering in the $0.47-\mu \mathrm{m}$ phase curve results. The isotropic $2 \mathrm{P}-\mathrm{HG}$ function 
predicts a highly forward scattering surface for both hemispheres at both wavelengths. This large difference is the result of no large phase angle measurements in the solar phase curve. Buratti (1991) found that the leading hemisphere of Callisto was more backscattering than the trailing hemisphere; however, her data set also did not include large phase angle observations for the leading hemisphere. Thus no conclusive statement can be made regarding hemispherical differences in the single scattering behavior on Callisto.

When interpreting the single particle scattering results a few caveats must be stated. McGuire and Hapke (1995) and Hartman and Domingue (1997) both demonstrated that the single scattering functions used in the analyses presented here are viable functions and well describe the laboratory scattering data of McGuire and Hapke. Therefore the significance between the isotropic $2 \mathrm{P}-\mathrm{HG}$ and $3 \mathrm{P}-$ HG results need to be explained.

The effects of a backward scattering component to the scattering function are most strongly seen between 0 and $40^{\circ}$ phase angle. Likewise, the effects of a forward scattering component to the scattering function are most strongly seen beyond $140^{\circ}$ phase angle. Since none of the phase curves analyzed here has measurements beyond $140^{\circ}$, the forward scattering component of the scattering function is not well constrained (see Error Analysis). However, these two scattering functions do not treat scattering in the same manner. The half-width of the forward and backward scattered components are assumed to be equivalent in the $2 \mathrm{P}$ HG function. For values of the relative amplitude parameter, $c_{2}$, midway between totally forward or totally backward scattering, the properties of the forward and backward scattered components are dependent on each other. This is not the case with the 3P-HG function, where there are two half-width parameters, one for each scattering component. Until there are good disk-integrated reflectance measurements beyond $140^{\circ}$ phase angle the nature of the scattering properties can only be estimated. Considering the phase angle coverage available in the phase curves presented here, the $2 \mathrm{P}-\mathrm{HG}$ solutions are better constrained.

\section{Anisotropic Multiple Scattering Results}

General. The RMS values of the anisotropic modeling results are within $0.5 \%$ of the isotropic modeling results, showing that statistically both fit the data equally well. However, the properties and shape of the single particle scattering function influence the values of the other parameters in Hapke's model. Below we discuss the different parameter values between the isotropic and anisotropic multiple scattering modeling results shown in Tables II and III in addition to examining the single particle scattering function predicted by both models.
Single scattering albedo. The values of the single scattering albedo in the isotropic versus the anisotropic multiple scattering model results for Europa are within $5 \%$. The isotropic model tends to produce slightly higher single scattering albedos. The modeling results for the darker surface of Ganymede show single scattering albedos within less than $1 \%$, which are well within the error bars. These results demonstrate that for a surface as bright as Europa the two models predict qualitatively similar single scattering albedos, but quantitatively the isotropic model produces slightly brighter estimates.

Roughness. Comparisons of the modeling results for the darker surface of Ganymede (as compared to Europa) show that both isotropic and anisotropic models predict the same macroscopic roughness properties to below $1 \%$ and well within the error bars. For the brighter surface of Europa, however, the differences in the predicted macroscopic surface roughness are slightly larger. Both models predict a much smoother surface as compared to Ganymede or Callisto, and are thus qualitatively similar. The quantitative differences are with the limits of the parameter error bars (for the $0.55-\mu \mathrm{m}$ Europa phase curve results the differences are at the edge of the error bars).

Opposition effect. One of the largest differences observed between the isotropic and anisotropic multiple scattering models is between the opposition effect parameters (bo and $h$ ). Even so, the solutions to Ganymede's phase curve have parameter values which are qualitatively similar and the differences are within the error bars for both opposition parameters. This is also true for the solutions to both $0.47-$ and $0.55-\mu \mathrm{m}$ leading phase curves of Europa. The phase curves for which the isotropic and anisotropic modeling results show differences outside of the estimated error bars are the Europa trailing hemisphere phase curves.

Single scattering function. Within the error bars the single particle scattering functions predicted by both isotropic and anisotropic models are the same for all analyses except for Europa's leading hemisphere. The results are plotted in Figs. 11 and 12. Phase angle coverage for this hemisphere extends from essentially $0^{\circ}$ to $120^{\circ}$. In this phase angle range the two models predict qualitatively similar single scattering behavior. The anisotropic solution predicts a slightly stronger backscattering component. At phase angles greater than $130^{\circ}$ the two models drastically diverge. The isotropic multiple scattering model predicts a substantial forward scattering component, whereas the anisotropic multiple scattering model predicts nearly no forward scattering behavior. There are no data beyond $120^{\circ}$ phase angle that would constrain the models in the forward scattering regimes. 


\section{DISCUSSION}

\section{Europa}

Europa has been observed to show a strong hemispherical dichotomy in its rotational lightcurve (Stebbins 1927, Stebbins and Jacobsen 1928, Millis and Thompson 1975, Morrison and Morrison 1977, Johnson et al. 1983, McEwen 1986, Nelson et al. 1986) where the leading hemisphere is brighter than the trailing hemisphere. McEwen (1986) showed that the hemispherical dichotomy could be modeled as a cosine function from the apex of orbital motion based on mosaics of the Voyager images, which is consistent with magnetospheric interactions with the surface (Pospieszalska and Johnson 1989). The hemispherical asymmetries on Europa have been attributed to two exogenic processes: (1) preferential bombardment of the leading hemisphere by micrometeorites (Shoemaker and Wolfe 1982, Clark et al. 1983, Clark and Lucey 1984) and (2) preferential sputtering by, and implantation of, magnetospheric ions on the trailing hemisphere (Lane et al. 1981, Clark et al. 1983, Johnson et al. 1984).

The main spectral component of Europa's surface is water ice (Pilcher et al. 1972) with only a few percent impurities (Clark 1980). Clark (1980) modeled the spectral characteristics of the icy Galilean satellites and found that Europa's spectrum most closely matches that of mediumto-fine grained water frost. Lane et al. (1981) reported the detection of an absorption feature at $0.28 \mu \mathrm{m}$ in the reflectance spectrum of Europa's trailing hemisphere taken with IUE, which they identified as an $\mathrm{SO}_{2}$ gas absorption feature. They hypothesized that this $\mathrm{SO}_{2}$ feature was a product of sulfur ion implanatation into a water ice matrix, providing evidence for the magnetospheric alteration of Europa's surface. More recently, however, Noll et al. (1995) made spectroscopic observations of Europa using the Hubble space telescope and identified a broad absorption feature at $0.28 \mu \mathrm{m}$ which they matched to $\mathrm{SO}_{2}$ frost, which is consistent with the laboratory work of Sack et al. (1992) and indicates a possible endogenic origin for the $\mathrm{SO}_{2}$ seen on Europa's trailing hemisphere. No clear detection of these absorption features has been made on Europa's leading hemisphere. Analysis of Europa's UV and visible spectra indicates the presence of sulfur dioxide and sulfur compounds (Lane et al. 1981, Sack et al. 1992, Calvin et al. 1995, Noll et al. 1995, Spencer et al. 1995); however, it is still debatable if the source is exogenic, endogenic, or a combination of these processes. Sack et al. (1991, 1992) showed that the general reddening in the UV of Europa's trailing hemisphere is not necessarily due to the implantation of sulfur or the deposition of sulfur dioxide, but is caused by the morphological changes in the ice grain structure from ion bombardment effects.

Sack et al. (1992) state that the ion bombardment of water ice causes an annealing effect which decreases the number of microcracks and bubbles which can act like internal scatterers. According to McGuire and Hapke (1995) and Hartman and Domingue (1997) particles with moderate to high densities of internal scatterers are more backscattering than those with little to no quantities of internal scatterers. Therefore single particle scattering functions which are more backscattering on the leading hemisphere than the trailing hemisphere are consistent with preferential ion bombardment on the trailing hemispheres. All modeling solutions presented in this paper show single particle scattering functions with backscattering components that are larger for the leading hemisphere solutions than the trailing hemisphere solutions. The extent of the forward scattering component appears to vary with hemisphere and with model. The isotropic multiple scattering 3P-HG model predicts a significant forward scattering component for the leading hemisphere and essentially little forward scattering on the trailing hemisphere. The anisotropic multiple scattering 3P-HG model, however, predicts essentially little forward scattering for both hemispheres. The isotropic and anisotropic 3P-HG models predict very similar scattering functions for Europa's trailing hemisphere, whereas the isotropic $2 \mathrm{P}-\mathrm{HG}$ model predicts a significant forward scattering component. The isotropic $2 \mathrm{P}$ HG and anisotropic 3P-HG solutions are similar for the leading hemisphere scattering functions, whereas the isotropic 3P-HG model predicts significant forward scattering. However, for the range of phase angles for which data are available, all three models show the same scattering behavior to wihin the parameter error bars. For the phase angle range for which coverage is available all three model solutions are consistent with the spectral characteristics of Europa's surface and the preferential ion bombardment of the trailing hemisphere. The deviations between the modeling results arise for those phase angles for which there are no data available to constrain the model parameters. Verbiscer and Veverka (1990), Verbiscer et al. (1990), and Domingue et al. (1997) have studied the scattering functions of terrestrial snow and have shown that regardless of the scattering function used in the modeling: terrestrial snows are forward scattering. Since water ice/frost is a main constituent of Europa's regolith, it is reasonable to hypothesize that the average scattering function of Europa's regolith may contain a forward scattering component. However, until very large $\left(>140^{\circ}\right)$ phase angle data are available the forward scattering nature of Europa's regolith may remain a mystery.

The values of the single scattering albedo for all model solutions are consistent with the lightcurves; the leading hemisphere values in both wavelengths are brighter than the trailing hemisphere values. The single scattering albedos from the isotropic $2 \mathrm{P}-\mathrm{HG}$ and isotropic $3 \mathrm{P}-\mathrm{HG}$ models are the same within the error bars. The anisotropic $3 \mathrm{P}$ HG model tends to predict slightly darker single scattering 
albedos than the isotropic 3P-HG model; however, the values are qualitatively similar. The small differences $(<0.03)$ are possibly a reflection of similarly small variations in other parameter values.

The low values of $\theta$-bar $\left(<15^{\circ}\right)$ found by all models indicate that the small roughness seen on the 2-km scale by the Voyager images extends down to the centimeter and millimeter scales. To within the error bars, the isotropic $2 \mathrm{P}-\mathrm{HG}$ and $3 \mathrm{P}-\mathrm{HG}$ models return similar values of the roughness parameter. The anisotropic 3P-HG model solutions show, in general, lower, and therefore smoother, surface roughness. One characteristic of the surface roughness parameter is that its value should be independent of wavelength; thus modeling solutions at 0.47 and $0.55 \mu \mathrm{m}$ should overlap within the roughness parameter error bar limits. This is true for the isotropic multiple scattering model solutions; however, this is not the case for the anisotropic 3P-HG model solutions for Europa's trailing hemisphere. The anisotropic modeling results for the surface roughness parameter solutions to the 0.47 - and $0.55-\mu \mathrm{m}$ phase curves do not overlap within the error bar limits.

The value of the opposition parameters indicates that both hemispheres are composed of a fairly transparent material (consistent with the $90 \%$ or greater water ice content estimated by Clark (1980)); however, the isotropic 3PHG solution values of bo indicate that the trailing hemisphere material is slightly more opaque, which is consistent with the observations of more dark material (possibly the sulfur and sulfur dioxide seen by Sack et al. (1992) and Noll et al. (1995)) being mixed with the water ice on the trailing hemisphere (sulfur dioxide frost is dark only in comparison to material with reflectance albedos of $80 \%$ or greater; Nelson et al. (1980)). The opposition width parameter from both isotropic multiple scattering models predicts a uniform particle size distribution and porosity on Europa's surface, which is consistent with a balance of ion bombardment on the trailing hemisphere and micrometeorite bombardment on the leading hemisphere. This contradicts the results of Buratti et al. (1988) who found the trailing hemisphere to be more porous than the leading hemisphere based on the analysis of IUE observations. The difference between the Buratti et al. (1988) findings and those in this paper (and Domingue et al. (1991)) is due to the absence of opposition measurements in their UV phase curves. The anisotropic 3P-HG model results for the opposition parameters are consistent with the isotropic results except for the solution values for Europa's $0.55-\mu \mathrm{m}$ trailing phase curve. The anisotropic multiple scattering solutions for the opposition parameters for this phase curve are equivalent to a more transparent paticle in a more compact regolith. However, the opposition width parameter $(h)$ measures surface properties which are independent of wavelength. Therefore model $h$ values should be independent of wavelength. This is true for the isotropic $2 \mathbf{P}$ -
HG and 3P-HG model solutions, but this is not the case for the anisotropic 3P-HG solutions for Europa's trailing hemisphere. Therefore the remainder of this discussion will focus on the isotropic multiple scattering model solutions.

Quantitative estimates of the surface porosity on Europa can be made assuming a particle grain size distribution function. The opposition width parameter $h$ is related to porosity by

$$
h=-\left(\frac{3}{8}\right) \ln (P) Y,
$$

where $P$ is the porosity (percentage of void space per unit volume) and $Y$ is the grain size distribution function. If a grain size distribution similar to the Moon's is assumed, then $Y$ is given by

$$
Y=\sqrt{3} / \ln \left(r_{1} / r_{\mathrm{s}}\right),
$$

where $r_{1}$ is the radius of the largest particle and $r_{s}$ is the radius of the smallest particle in the size distribution (Hapke 1986). Figure 14 plots the porosity of Europa's surface as a function of $r_{1} / r_{\mathrm{s}}$. The porosities of the other icy Galilean satellites made using the same assumptions are plotted for comparison along with the results from Hartman and Domingue (1997) for the Moon. The high porosity for Europa is consistent with a medium to finegrained water frost.

\section{Ganymede}

Ganymede also displays a hemispherical dichotomy in its visible lightcurve which is in the same sense as Europa but not as pronounced (Stebbins 1927, Stebbins and Jacobsen 1928, Millis and Thompson 1975, Morrison and Morrison 1977, Johnson et al. 1983). The exogenic processes at work on Europa are also proposed to be the sources of the hemispherical differences on Ganymede.

Water ice has also been identified on the surface of Ganymede (Pilcher et al. 1972), with a larger fraction of impurities present than estimated on Europa. If the water ice is intimately mixed with the darker non-ice component, then the estimated water ice abundance is on the order of 90 wt\% (Clark 1980); however, areal mixing models show that the water ice coverage on Ganymede could be as low as $50 \%$ and still agree with the spectral data (Spencer 1987). The best fits to Ganymede's spectra show that the water absorption bands are best modeled as frost on ice (Clark 1980) with the frost grain size larger than those predicted for Europa (Clark 1980, Clark et al. 1986, Calvin et al. 1995). The spectra also indicate that the grain sizes on Ganymede's trailing hemisphere are larger than those on the leading hemisphere (Clark et al. 1986, Calvin et al. 1995). In comparing Ganymede's spectra with Europa's, Nelson et al. (1987) noticed an additional source of absorp- 


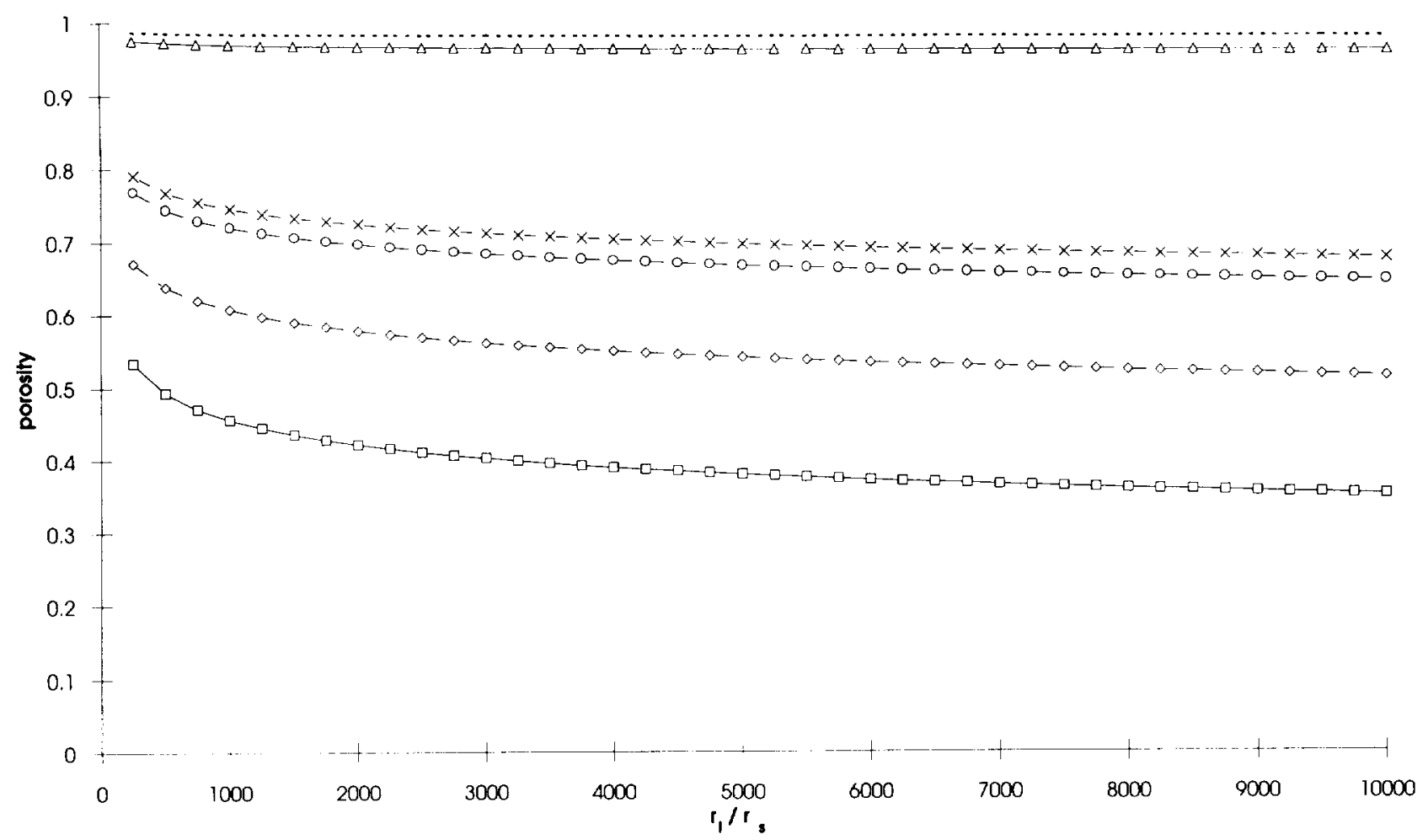

FIG. 14. Porosity profiles for Europa (dashed line), Ganymede's leading (triangles) and trailing (squares) hemispheres, Callisto's leading (circles) and trailing (crosses) hemispheres, and the lunar near side (diamonds). These porosity values are given as a function of the ratio of the radii of the largest to smallest particle of the regolith assuming a grain size distribution typical of comminution processes.

tion in Ganymede's trailing hemisphere spectra shortward of $0.28 \mu \mathrm{m}$ which they attributed to the presence of molecular oxygen in the form of ozone. Noll et al. (1996) observed both hemispheres of Ganymede in the UV with the Hubble space telescope and detected the presence of an absorption band on Ganymede's trailing hemisphere at $0.26 \mu \mathrm{m}$ which they identified as the Hartley band of ozone. This absorption band was not present on the leading hemisphere. Both Nelson et al. (1987) and Noll et al. (1996) attribute the presence of ozone on the trailing hemisphere to ion bombardment and implantation process. Spencer et al. (1995) and Calvin et al. (1995) report the detection of absorption features in the visible at 0.577 and $0.627 \mu \mathrm{m}$ due to the presence of solid diatomic oxygen on Ganymede's trailing hemisphere, which is consistent with the preferential interaction of the magnetosphere with the trailing hemisphere.

All model solutions are consistent with the spectral observations of Ganymede's surface structure and characteristics. They all show that the single scattering albedo is darker on Ganymede than Europa, which is consistent with geometric albedo measurements of these two satellites. Both isotropic multiple scattering models find that the single scattering albedo at $0.55 \mu \mathrm{m}$ is brighter on the leading hemisphere than the trailing hemisphere (which is consis- tent with the visible lightcurves); however both models show that the opposite is true at $0.47 \mu \mathrm{m}$, which is unexpected. The anisotropic multiple scattering model was not fit to the $0.55-\mu \mathrm{m}$ data (due to lack of phase angle data at larger, $>90^{\circ}$, phase angles); therefore similar comparisons cannot be made. A wavelength dependence is seen in some of the model solutions. The trailing hemisphere at $0.47 \mu \mathrm{m}$ has a nearly isotropic scattering function predicted by the models, whereas at $0.55 \mu \mathrm{m}$ the solutions show a strongly backscattering behavior. Each model predicts a backscattering behavior for Ganymede's leading hemisphere at 0.47 $\mu \mathrm{m}$. However, the isotropic $2 \mathrm{P}-\mathrm{HG}$ model predicts a forward scattered component at $0.55 \mu \mathrm{m}$.

The roughness dichotomy measured by all models agrees within their error bars. A rougher trailing hemisphere is also consistent with a lower physical albedo on the trailing hemisphere. It is noted that the $0.55-\mu \mathrm{m}$ phase curves for both hemispheres of Ganymede lack the necessary large phase angle observations to make realistic determinations of the Hapke roughness parameter. However, the agreement with the $0.47-\mu \mathrm{m}$ Hapke roughness parameters lends validity to the $0.55-\mu \mathrm{m}$ roughness measurements since the roughness parameter does not vary with wavelength.

The opposition parameter values of all the models are 
consistent with the evidence that there is a larger percentage of non-ice component on Ganymede compared to Europa. The bo values for Ganymede at $0.47 \mu \mathrm{m}$ indicate that more opaque material is present on Ganymede than on Europa; however, the $0.55-\mu \mathrm{m}$ results give conflicting measurements. The spectral work of Clark (1980), Clark et al. (1986), and Calvin et al. (1955) estimates that the particle grain sizes on Ganymede are generally larger than those on Europa, smaller than those on Callisto, and have the added complication that they are larger on the trailing hemisphere than the leading hemisphere. The opposition width parameter is physically related to both porosity and grain size distribution. The smaller values of $h$ are generally correlated to larger porosities or small compaction states; however the correlation with grain size is less straightforward since it is more strongly dependent on the grain size distribution (the ratio of largest grain to smallest grain). If smaller values of $h$ are correlated with smaller grain sizes, then the spectral analyses would predict that the values of $h$ in increasing order would be Europa's surface (and possibly the leading hemisphere of Callisto), the leading hemisphere of Ganymede, the trailing hemisphere of Ganymede, and the trailing hemisphere of Callisto. The Hapke model solutions show that the only $h$ values which do not follow this trend are the values for Ganymede's trailing hemisphere and Callisto's leading hemisphere. This trend, however, assumes similar porosities and that smaller grain sizes correspond to smaller grain size distributions, which are extremely unlikely. The plots in Fig. 14 compare the porosity estimates from the isotropic multiple scattering solutions of both hemispheres of Ganymede assuming a lunar-like particle distribution. The anisotropic multiple scattering solutions are similar to the isotropic multiple scattering modeling results and are, therefore, not included. The porosity of the leading hemisphere is very similar to Europa's surface; however, the trailing hemisphere is more compact than the lunar regolith. This compaction state is highly unlikely in view of the match between Ganymede's spectra and that of frost on ice. This indicates that the grain size distribution function assumed in the derivation of these porosities is not indicative of the grain size distribution on the surface of Ganymede.

\section{Callisto}

Callisto displays a hemispherical dichotomy in its visible lightcurve which is in the opposite sense to that seen on Europa and Ganymede (Stebbins 1927, Stebbins and Jacobsen 1928, Millis and Thompson 1975, Morrison and Morrison 1977, Johnson et al. 1983); i.e., the trailing hemisphere is brighter than the leading hemisphere. Buratti (1991) and Calvin and Clark (1993) conclude from their analyses that the dominant process affecting the optical surface of Callisto is micrometeoritic gardening. Meteorite bombardment is thought to be the dominant exogenic process at work on Callisto since its orbit is distant enough to minimize magnetospheric interaction effects.

Water ice absorptions have also been detected on Callisto (Pilcher et al. 1972). However, it has the largest amount of dark non-ice component incorporated into its surface among the three icy Galilean satellites. Clark (1980) estimated that the amount of water on Callisto ranges from 30 to $90 \mathrm{wt} \%$ assuming an intimate mixture of ice and non-ice component. In contrast, modeling studies by Calvin and Clark (1991) and Roush et al. (1990) showed that the weight percent of water on Callisto could be as low as 20 to $45 \%$ if combinations of intimate and areal mixtures of the water ice and non-ice components are considered. Spencer (1987) showed that areal mixtures of $10 \%$ water ice are consistent with the spectral data. The water ice grain size on Callisto is the largest of the three satellites, being predominately coarse grained (Calvin and Clark 1991, Roush et al. 1990). Calvin and Clark (1993) and Calvin et al. (1995) noted, however, that there is a water ice grain size dichotomy on Callisto, with the spectral signature of fine grained water ice in the 3- to $3.6-\mu \mathrm{m}$ region matching that of the leading hemisphere.

The largest differences in surface structure predicted by the isotropic $2 \mathrm{P}-\mathrm{HG}$ and $3 \mathrm{P}-\mathrm{HG}$ models are for the surface of Callisto. The values of the single scattering albedo found with the isotropic 3P-HG model are consistent with the visible lightcurves; the trailing hemisphere values in both wavelengths are higher than the leading hemisphere values. The isotropic $2 \mathrm{P}-\mathrm{HG}$ model shows the opposite trend which is inconsistent with the lightcurves. If the observations of a more dark, non-ice component on Callisto than on the other two icy Galilean satellites are accurate, then the bo values returned by the models should be higher for Callisto, indicative of the presence of more opaque material. Both models are consistent with this predicted trend with the anomalous exception of the $0.47-\mu \mathrm{m}$ solutions for trailing hemisphere. The values of the opposition width parameter, $h$, are consistent with a more porous trailing hemisphere. The spectral evidence suggests that the grain sizes on the trailing hemisphere are larger than those on the leading hemisphere, which may be similar in grain size to that seen in Europa's surface. This would imply a grain size distribution on the trailing hemisphere smaller than that on the leading hemisphere, thus raising the possibility of a higher small grain frost content on the leading hemisphere. The porosities plotted in Fig. 14 are consistent with this scenario. Without any large phase angle data for the leading hemisphere any discussion of hemispherical differences in surface roughness or single particle scattering function is speculation only. 


\section{CONCLUSIONS}

The contrasts between our results and those of Buratti (1991) for Ganymede and Callisto indicate that there is a vast difference in the predicted physical characteristics of a planetary regolith dependent on the choice of single particle scattering function incorporated in Hapke's model. The results presented here show that the general physical characteristics predicted by a Hapke model (with isotropic multiple scattering) incorporating a $2 \mathrm{P}$-HG scattering function are similar to the predictions given by a similar Hapke model incorporating a $3 \mathrm{P}-\mathrm{HG}$ function as long as the data set being modeled has adequate coverage in phase angle. Conflicting results occur when the large angle coverage is inadequate. For surfaces as bright or darker than Ganymede both isotropic and anisotropic models predict similar photometric behavior over the phase angle ranges of the data set. For phase curves with phase angle coverage less than $120^{\circ}$ the isotropic and anisotropic models predict similar results (within that limited phase angle range) for objects as bright as Europa; however, they may predict different single scattering behavior for phase angle ranges not included in or constrained by observations. The photometric surface textures predicted in our results support the spectral evidence for magnetospheric alterations of the optical properties of the surface of Europa and Ganymede.

\section{ACKNOWLEDGMENTS}

D. Domingue thanks the following people for their valued assistance: G. Hansen, B. Hartman, A. Kubala, and B. Fessler. D. Domingue and A. Vehiscer thank John Spencer and Robert Nelson for insightful reviews and constructive comments. A. Verbiscer acknowledges the support of NASA Grant NAGW-3591. LPI Contribution 920.

\section{REFERENCES}

Allen, C. W. 1973. Astrophysical Quantities. Athlone Press, UK.

Bevington, P. R. 1969. Data Reduction and Error Analysis for the Physical Sciences. McGraw-Hill. New York.

Buratti, B. 1985. Application of a radiative transfer model to bright icy satellites. Icarus 61, 208-217.

Buratti, B. 1991. Ganymede and Callisto: Surface textural dichotomies and photometric analysis. Icarus 92, 312-323.

Buratti. B., R. M. Nelson, and A. L. Lane 1988. Surficial textures of the Galilean satellites. Noture (London) 33, 148-151.

Burns, J. A. 1986. Satellites (J. A. Burns and M. S. Matthews, Eds.), pp 1-38. Univ. of Arizona Press, Tucson.

Calvin. W. M., and R. N. Clark 1993. Spectral distinctions between the leading and trailing hemispheres of Callisto: New observations. Icaris $104,69-78$

Calvin, W. M.. R. N. Clark. R. H. Brown, and J. R. Spencer 1995. Spectra of the ice Galilean satellites from 0.2 to $5 \mu \mathrm{m}$ : A compilation, new observations, and a recent summary. J. Geophys Res. 100, 19141-19048.

Clark. R. 1980. Ganymede. Europa. Callisto. Saturn's rings: Compositional analysis for reflectance spectroscopy. Icarus 44, 388-409.
Clark, R. N.. and P. G. Lucey 1984. Spectral properties of ice-particulate mixtures and implications for remote sensing. 1 . Intimate mixtures. $J$. Geophys. Res. 89, 6341-6.348.

Clark. R. N., F. P. Fanale, and M. J. Gaffey 1986. Surface composition of satellites. In Satellites (J. Burns and M. S. Matthews, Eds.). Univ. of Arizona Press, Tucson.

Clark, R. N., R. P. Fanale, and A. P. Zent 1983. Frost grain size metamorphism: Implications for remote sensing of planetary surfaces. Icarus 56, 233-245.

Danielson, G. E.. P. N. Kupferman, T. V. Johnson, and L. A. Soderblom 1981. Radiometric performance of the Voyager cameras. I. Geophys. Res. 86, 8683-8689.

Domingue, D. L., B. W. Hapke, G. W. Lockwood. and D. T. Thompson 1991. Europa a phase curve: Implications for surface structure. Icarus $90,30-42$.

Domingue, D., B. Hartman, and A. Verbiscer 1997. The scattering properties of natural terrestrial snows versus icy satellite surfaces. Larm. $128,28-48$.

Goguen, J. D. 1996. A quantitative test of the applicability of independent scattering to high albedo planetary regoliths. lcarus, submitted.

Hapke, B. 1981. Bidirectional reflectance spectroscopy. 1. Theory. I. Geophys. Res. 86, 30.39-3054.

Hapke, B. 1984. Bidirectional reflectance spectroscopy. 3. Correction for macroscopic roughness. Icarus 59, 41-59.

Hapke, B. 1986. Bidirectional reflectance spectroscopy. 4. The extinction coefficient and the opposition effect. Icarts. 67, 264-280.

Hartman. B., and D. Domingue 1997. Scatlering of light by individual particles and the implications for models of planctary surfaces. Icarus. in press.

Helfenstein. P. 1986. Derivation and Analysis of Geological Constraints on the Emplacemont and Evolution of Terrains on Ganymede from Applied Differential Photometry. Thesis. Brown Univ.

Helfenstein, P., J. Hillier, and J. Veverka 1997. Albedo dependence of particle phase functions for planctary regoliths. Karus, subnitled.

Johnson. R. E., L. A. Barton, J. W. Jesser. W. L. Brown. and L. J. Lanzerotti 1984. In Ites in the Solar System (J. Klinger. Ed.). pp. 301315. Reidel, Holland.

Johnson, T. V., L. A. Soderblom, J. A. Mosher, G. H. Danielson, A. F. Cook, and P. Kupferman 1983. Global multispectral mosaics of the icy Galilean satcllites. J. Geophys. Res. 88, 5789-5805.

Lane, A. L., R. M. Nelson, and D. L. Matson 1981. Evidence for sulfur implantation in Europa's UV absorption band. Nafure (London) $292,38-39$.

McEwen. A. 1986. Exogenic and endogenic alhedo and color patterns on Europa. J. Geophys. Res. B8, 8077-8097.

McGuire, A. F., and B. W. Hapke 1995. An experimental study of light scattering by large, irregular particles. lcarus 113, 1.34-135.

Millis. R. L.. and D. T. Thompson 1975. UBV photometry of the Galilean satellites. Icarus 24, 406-419.

Mishchenko, M. 1. 1994. Asymmetry parameters of the phase function for densely packed scattering grains. J. Quamt. Spectrosc. Radiar. Transfer, 52, No. 1. 95-110.

Morrison. D., and N. Morrison 1977. Photomerry of the Galilean Sarellites, Planerary Satellites (J. A. Burns. Ed.). pp. 363-378. Univ. of Arizona Press, Tucson.

Morrison. D. N., N. Morrison, and A. Lazarewicz 1974. Four-color photometry of the Galilean satellites. larus 23, 399-416.

Nelson. M. L., T. B. McCord. R. N. Clark. T. V. Johnson. D. L. Matson, J. A. Mosher. and L. A. Soderblom 1986. Europa: Characterization and interpretation of global spectral surface units. Icames 65, 129-151. 
Nelson, R. M., A. L. Lane, D. L. Matson, F. P. Fanale, D. B. Nash, and T. V. Johnson 1980. Io: Longitudinal distribution of sulfur dioxide frost. Science 210, 784-786.

Nelson, R. M., A. L. Lane, D. L. Matson, G. J. Veeder, B. J. Buratti, and E. F. Tedesco 1987. Spectral geometric albedos of the Galilean satellites from 0.24 to 0.34 micrometers: Observations with the International Ultraviolet Explorer. Icarus 72, 358-380.

Noll, K. S., R. Johnson, A. L. Lane, D. Domingue, and H. A. Weaver 1996. Detection of ozone on Ganymede. Science 273, 341-343.

Noll, K. S., H. A. Weaver, and A. M. Gonnella 1995. The albedo spectrum of Europa from 2201$) \AA$ to $3300 \AA$. . J. Geophys. Res. 100, 19057-19059.

Pilcher, C. B., S. T. Ridgeway, and T. B. McCord 1972. Galilean satellites: Identification of water frost. Science 178, 1087-1089.

Pospieszalska, M. K., and R. E. Johnson 1989. Magnetospheric ion bombardment profiles of satellites: Europa and Dione. Icarus 78, 1-13.

Roush, T. L., J. B. Pollack, F. C. Witteborn, J. D. Bregman, and J. P. Simpson 1990. Ice and minerals on Callisto: A reassessment of the reflectance spectra. Icarus 86, 355-382.

Sack, N. J., J. W. Boring, R. E. Johnson, R. A. Baragiola, and M. Shi 1991. Alteration of the UV-visible reflectance spectra of $\mathrm{H}_{2} \mathrm{O}$ ice by ion bombardment. J. Geophys Res. Planets E96(2), 17535-17539.

Sack, N. J., R. E. Johnson. J. W. Boring, and R. A. Baragiola 1992. The effect of magnetospheric ion bombardment on the reflectance of Europa's surface. Icarus 100, 534-540.
Shoemaker, E. M., and R. F. Wolfe 1982. Satellites of Jupiter (D. Morrison, Ed.), pp. 277-339. Univ. of Arizona Press, Tucson.

Spencer, J. R. 1987. Icy Galilean satellite reflectance spectra: Less ice on Ganymede and Callisto? Icarus 70, 99-110.

Spencer, J. R., W. M. Calvin, and M. J. Person 1995. Charge-coupled device spectra of the Galilean satellites: Molecular oxygen on Ganymede. J. Geophys. Res. 100, 19049-19056.

Stebbins, J. 1927. The light-variations of the satellites of Jupiter and their application to measures of the solar constant. Lick Obs. Bull. 13, $1-11$.

Stebbins, J, and T. S. Jacobsen 1928. Further photometric measures of Jupiter's satellites and Uranus, with tests for the solar constant. Lick Obs. Bull. 13, 180-195.

Verbiscer, A. 1991. Photometry of Icy Satellite Surfaces. Ph.D. thesis, Cornell University, Ithaca, NY.

Verbiscer, A., and P. Helfenstein 1997. Reflectance spectroscopy of icy surfaces. In Ices in the Solar System (B. Schmitt, C. deBergh, and M. Festou, Eds.). Kluwer, in press.

Verbiscer, A. J., and J. Veverka 1990 . Scattering properties of natural snow and frost: Comparison with ice satellite photometry. Icarus $\mathbf{8 8}$, $418-428$.

Verbiscer, A., P. Helfenstein, and J. Veverka 1990. Icy satellites in the outer solar system: A backscattering frost component? Nature $\mathbf{3 4 7}$, 162-164. 